\title{
Systems of Structured Monotone Inclusions: Duality, Algorithms, and Applications*
}

\author{
Patrick L. Combettes \\ UPMC Université Paris 06 \\ Laboratoire Jacques-Louis Lions - UMR CNRS 7598 \\ 75005 Paris, France \\ plc@math.jussieu.fr
}

\begin{abstract}
A general primal-dual splitting algorithm for solving systems of structured coupled monotone inclusions in Hilbert spaces is introduced and its asymptotic behavior is analyzed. Each inclusion in the primal system features compositions with linear operators, parallel sums, and Lipschitzian operators. All the operators involved in this structured model are used separately in the proposed algorithm, most steps of which can be executed in parallel. This provides a flexible solution method applicable to a variety of problems beyond the reach of the state-of-the-art. Several applications are discussed to illustrate this point.
\end{abstract}

Keywords convex minimization, coupled system, infimal convolution, monotone inclusion, monotone operator, operator splitting, parallel algorithm, structured minimization problem

Mathematics Subject Classifications (2010) Primary 47H05; Secondary 65K05, 90C25.

\section{Introduction}

Traditional monotone operator splitting techniques [8, 17, 23, 24, 28, 34, 36, 40, 42, 43] have their roots in matrix decomposition methods in numerical analysis [21, 44] and in nonlinear methods for solving optimization and variational inequality problems [7, 11, 30, 33, 39]. These methods are designed to solve inclusions of the type $0 \in B_{1} x+B_{2} x$, where $B_{1}$ and $B_{2}$ are maximally monotone operators acting on a Hilbert space $\mathcal{H}$. Extensions to sums of the type $0 \in \sum_{k=1}^{K} B_{k} x$ are typically handled via reformulations in product spaces [8, 40]. In recent years, new splitting algorithms have emerged for problems involving more complex models featuring compositions with linear operators [13] and parallel sums [19, 45] (see (1.9)). These algorithms rely on reformulations of the inclusions as two-operator problems in a primal-dual space, in which the splitting is performed via an existing method. This construct makes it possible to activate separately each of the operators

${ }^{*}$ Contact author: P. L. Combettes, plc@math.jussieu.fr, phone: +33 14427 6319, fax: +33 144277200. 
present in the model, and it leads to flexible algorithms implementable on parallel architectures. In the present paper, we pursue this strategy towards more sophisticated models involving systems of structured coupled inclusions in duality. The primal-dual problem under consideration is the following.

Problem 1.1 Let $m$ and $K$ be strictly positive integers, let $\left(\mathcal{H}_{i}\right)_{1 \leqslant i \leqslant m}$ and $\left(\mathcal{G}_{k}\right)_{1 \leqslant k \leqslant K}$ be real Hilbert spaces, let $\left(\mu_{i}\right)_{1 \leqslant i \leqslant m} \in\left[0,+\infty\left[^{m}\right.\right.$, and let $\left(\nu_{k}\right)_{1 \leqslant i \leqslant K} \in\left[0,+\infty\left[^{K}\right.\right.$. For every $i \in\{1, \ldots, m\}$ and $k \in$ $\{1, \ldots, K\}$, let $C_{i}: \mathcal{H}_{i} \rightarrow \mathcal{H}_{i}$ be monotone and $\mu_{i}$-Lipschitzian, let $A_{i}: \mathcal{H}_{i} \rightarrow 2^{\mathcal{H}_{i}}$ and $B_{k}: \mathcal{G}_{k} \rightarrow 2^{\mathcal{G}_{k}}$ be maximally monotone, let $D_{k}: \mathcal{G}_{k} \rightarrow 2^{\mathcal{G}_{k}}$ be maximally monotone and such that $D_{k}^{-1}: \mathcal{G}_{k} \rightarrow \mathcal{G}_{k}$ is $\nu_{k}$-Lipschitzian, let $z_{i} \in \mathcal{H}_{i}$, let $r_{k} \in \mathcal{G}_{k}$, and let $L_{k i} \in \mathcal{B}\left(\mathcal{H}_{i}, \mathcal{G}_{k}\right)$. It is assumed that

$$
\beta=\max \left\{\max _{1 \leqslant i \leqslant m} \mu_{i}, \max _{1 \leqslant k \leqslant K} \nu_{k}\right\}+\sqrt{\lambda}>0, \quad \text { where } \quad \lambda \in\left[\sup _{\sum_{i=1}^{m}\left\|x_{i}\right\|^{2} \leqslant 1} \sum_{k=1}^{K}\left\|\sum_{i=1}^{m} L_{k i} x_{i}\right\|^{2},+\infty[,\right.
$$

and that the system of coupled inclusions

find $\overline{x_{1}} \in \mathcal{H}_{1}, \ldots, \overline{x_{m}} \in \mathcal{H}_{m}$ such that

$$
\left\{\begin{aligned}
z_{1} & \in A_{1} \overline{x_{1}}+\sum_{k=1}^{K} L_{k 1}^{*}\left(\left(B_{k} \square D_{k}\right)\left(\sum_{i=1}^{m} L_{k i} \overline{x_{i}}-r_{k}\right)\right)+C_{1} \overline{x_{1}} \\
& \vdots \\
z_{m} & \in A_{m} \overline{x_{m}}+\sum_{k=1}^{K} L_{k m}^{*}\left(\left(B_{k} \square D_{k}\right)\left(\sum_{i=1}^{m} L_{k i} \overline{x_{i}}-r_{k}\right)\right)+C_{m} \overline{x_{m}}
\end{aligned}\right.
$$

possesses at least one solution. Solve (1.2) together with the dual problem

find $\overline{v_{1}} \in \mathcal{G}_{1}, \ldots, \overline{v_{K}} \in \mathcal{G}_{K}$ such that

$$
\left\{\begin{aligned}
-r_{1} & \in-\sum_{i=1}^{m} L_{1 i}\left(A_{i}+C_{i}\right)^{-1}\left(z_{i}-\sum_{k=1}^{K} L_{k i}^{*} \overline{v_{k}}\right)+B_{1}^{-1} \overline{v_{1}}+D_{1}^{-1} \overline{v_{1}} \\
& \vdots \\
-r_{K} & \in-\sum_{i=1}^{m} L_{K i}\left(A_{i}+C_{i}\right)^{-1}\left(z_{i}-\sum_{k=1}^{K} L_{k i}^{*} \overline{v_{k}}\right)+B_{K}^{-1} \overline{v_{K}}+D_{K}^{-1} \overline{v_{K}} .
\end{aligned}\right.
$$

The primal system (1.2) captures a broad class of problems in nonlinear analysis in which $m$ variables $x_{1}, \ldots, x_{m}$ interact. The $i$ th inclusion in (1.2) features two operators $A_{i}$ and $C_{i}$ which model some abstract utility of the variable $x_{i}$, while the operator $\left(B_{k}\right)_{1 \leqslant k \leqslant K},\left(D_{k}\right)_{1 \leqslant k \leqslant K}$, and $\left(L_{k i}\right)_{\substack{1 \leqslant i \leqslant m \\ 1 \leqslant k \leqslant K}}$ model the interaction between $x_{i}$ and the remaining variables. One of the simplest realizations of (1.2) is the problem considered in [10], namely

$$
\text { find } \overline{x_{1}} \in \mathcal{H}, \overline{x_{2}} \in \mathcal{H} \text { such that }\left\{\begin{array}{l}
0 \in A_{1} \overline{x_{1}}+\overline{x_{1}}-\overline{x_{2}} \\
0 \in A_{2} \overline{x_{2}}-\overline{x_{1}}+\overline{x_{2}},
\end{array}\right.
$$

where $(\mathcal{H},\|\cdot\|)$ is a real Hilbert space, and where $A_{1}$ and $A_{2}$ are maximally monotone operators acting on $\mathcal{H}$. In particular, if $A_{1}=\partial f_{1}$ and $A_{2}=\partial f_{2}$ are the subdifferentials of proper lower semicontinuous convex functions $f_{1}$ and $f_{2}$ from $\mathcal{H}$ to $]-\infty,+\infty$ ], (1.4) becomes

$$
\underset{x_{1} \in \mathcal{H}, x_{2} \in \mathcal{H}}{\operatorname{minimize}} f_{1}\left(x_{1}\right)+f_{2}\left(x_{2}\right)+\frac{1}{2}\left\|x_{1}-x_{2}\right\|^{2} .
$$


This formulation arises in areas such as optimization [1], the cognitive sciences [5], image recovery [20], signal synthesis [29], best approximation [9], and mechanics [37]. In [3], we considered the extension of (1.5) which amounts to setting in Problem 1.1, for every $i \in\{1, \ldots, m\}$ and $k \in\{1, \ldots, K\}, A_{i}=\partial f_{i}, C_{i}=0$, and $B_{k}=\nabla g_{k}$, where $\left.\left.f_{i}: \mathcal{H} \rightarrow\right]-\infty,+\infty\right]$ is a proper lower semicontinuous convex function and $g_{k}: \mathcal{G}_{k} \rightarrow \mathbb{R}$ is convex and differentiable with a Lipschitzian gradient. This leads to the minimization problem

$$
\underset{x_{1} \in \mathcal{H}_{1}, \ldots, x_{m} \in \mathcal{H}_{m}}{\operatorname{minimize}} \sum_{i=1}^{m} f_{i}\left(x_{i}\right)+\sum_{k=1}^{K} g_{k}\left(\sum_{i=1}^{m} L_{k i} x_{i}\right),
$$

which has numerous applications in signal processing, machine learning, image recovery, partial differential equations, and game theory; see [2, 6, 12, 14, 25, 27, 41] and the references therein. In the case when $m=1$ in Problem 1.1, and under certain restrictions on the operators involved, primal-dual algorithms have been proposed recently in [13, 19, 45]. On the other hand, a primal algorithm was proposed in [3] for solving systems of inclusions of type (1.2) in which the operators $\left(C_{i}\right)_{1 \leqslant i \leqslant m}$ and $\left(D_{k}^{-1}\right)_{1 \leqslant k \leqslant K}$ are zero, and the coupling operators $\left(B_{k}\right)_{1 \leqslant k \leqslant K}$ are restricted to be single-valued and to satisfy jointly a cocoercivity property.

The goal of the present paper is to develop a flexible algorithm to solve Problem 1.1 without the restrictions imposed by current methods. In particular, no additional hypotheses will be placed neither on the coupling operators $\left(B_{k}\right)_{1 \leqslant k \leqslant K}$ and $\left(D_{k}\right)_{1 \leqslant k \leqslant K}$, nor on the number $m$ of variables. In the proposed parallel splitting algorithm, the structure of the problem is fully exploited to the extent that the operators are all used individually, either explicitly if they are single-valued, or by means of their resolvent if they are set-valued. The main algorithm is introduced and analyzed in Section 2, The remaining sections are devoted to applications to problems which are not explicitly solvable via existing techniques. Thus, in Section 3, we discuss applications to univariate inclusion problems featuring general parallel sums, in the sense that the operators $\left(D_{k}\right)_{1 \leqslant k \leqslant K}$ need not have Lipschitzian inverses. In Section 4, we apply this framework to the regularization of inconsistent common zero problems. Finally, Sections 5 and 6 address, respectively, applications to multivariate and univariate structured convex minimization problems.

Notation. We denote the scalar product of a Hilbert space by $\langle\cdot \mid \cdot\rangle$ and the associated norm by $\|\cdot\|$. The symbols $\rightarrow$ and $\rightarrow$ denote, respectively, weak and strong convergence, and Id denotes the identity operator. Let $\mathcal{H}$ and $\mathcal{G}$ be real Hilbert spaces and let $2^{\mathcal{H}}$ be the power set of $\mathcal{H}$. The space of bounded linear operators from $\mathcal{H}$ to $\mathcal{G}$ is denoted by $\mathcal{B}(\mathcal{H}, \mathcal{G})$. Let $A: \mathcal{H} \rightarrow 2^{\mathcal{H}}$. We denote by $\operatorname{ran} A=\{u \in \mathcal{H} \mid(\exists x \in \mathcal{H}) u \in A x\}$ the range $A$, by $\operatorname{dom} A=\{x \in \mathcal{H} \mid A x \neq \varnothing\}$ the domain of $A$, by zer $A=\{x \in \mathcal{H} \mid 0 \in A x\}$ the set of zeros of $A$, by $\operatorname{gra} A=\{(x, u) \in \mathcal{H} \times \mathcal{H} \mid u \in A x\}$ the graph of $A$, and by $A^{-1}$ the inverse of $A$, i.e., the operator with graph $\{(u, x) \in \mathcal{H} \times \mathcal{H} \mid u \in A x\}$. The resolvent of $A$ is $J_{A}=(\mathrm{Id}+A)^{-1}$. Moreover, $A$ is declared monotone if

$$
(\forall(x, u) \in \operatorname{gra} A)(\forall(y, v) \in \operatorname{gra} A) \quad\langle x-y \mid u-v\rangle \geqslant 0,
$$

and maximally monotone if there exists no monotone operator $B: \mathcal{H} \rightarrow 2^{\mathcal{H}}$ such that gra $A \subset$ $\operatorname{gra} B \neq \operatorname{gra} A$. In this case, $J_{A}$ is a nonexpansive operator defined everywhere on $\mathcal{H}$. Furthermore, $A$ is uniformly monotone at $x \in \operatorname{dom} A$ if there exists an increasing function $\phi:[0,+\infty[\rightarrow[0,+\infty]$ that vanishes only at 0 such that

$$
(\forall u \in A x)(\forall(y, v) \in \operatorname{gra} A) \quad\langle x-y \mid u-v\rangle \geqslant \phi(\|x-y\|),
$$

and $A$ is couniformly monotone at $u \in \operatorname{ran} A$ if $A^{-1}$ is uniformly monotone at $u$. The parallel sum of $A$ and $B: \mathcal{H} \rightarrow 2^{\mathcal{H}}$ is

$$
A \square B=\left(A^{-1}+B^{-1}\right)^{-1} \text {. }
$$


The infimal convolution of two functions $g$ and $\ell$ from $\mathcal{H}$ to $]-\infty,+\infty]$ is

$$
g \square \ell: \mathcal{H} \rightarrow[-\infty,+\infty]: x \mapsto \inf _{y \in \mathcal{H}}(g(y)+\ell(x-y)) .
$$

We denote by $\Gamma_{0}(\mathcal{H})$ the class of lower semicontinuous convex functions $\left.f: \mathcal{H} \rightarrow\right]-\infty,+\infty$ ] such that $\operatorname{dom} f=\{x \in \mathcal{H} \mid f(x)<+\infty\} \neq \varnothing$. Let $f \in \Gamma_{0}(\mathcal{H})$. The conjugate of $f$ is $\Gamma_{0}(\mathcal{H}) \ni f^{*}: u \mapsto$ $\sup _{x \in \mathcal{H}}(\langle x \mid u\rangle-f(x))$, and $f$ is uniformly convex at $x \in \operatorname{dom} f$ if there exists an increasing function $\phi:[0,+\infty[\rightarrow[0,+\infty]$ that vanishes only at 0 such that

$$
(\forall y \in \operatorname{dom} f)(\forall \alpha \in] 0,1[) \quad f(\alpha x+(1-\alpha) y)+\alpha(1-\alpha) \phi(\|x-y\|) \leqslant \alpha f(x)+(1-\alpha) f(y) .
$$

For every $x \in \mathcal{H}, f+\|x-\cdot\|^{2} / 2$ possesses a unique minimizer, which is denoted by $\operatorname{prox}_{f} x$. We have

$$
\operatorname{prox}_{f}=J_{\partial f}, \quad \text { where } \quad \partial f: \mathcal{H} \rightarrow 2^{\mathcal{H}}: x \mapsto\{u \in \mathcal{H} \mid(\forall y \in \mathcal{H})\langle y-x \mid u\rangle+f(x) \leqslant f(y)\}
$$

is the subdifferential of $f$. Let $C$ be a convex subset of $\mathcal{H}$. The indicator function of $C$ is denoted by $\iota_{C}$ and the distance function to $C$ by $d_{C}$. The relative interior [respectively, the strong relative interior] of $C$, i.e., the set of points $x \in C$ such that the cone generated by $-x+C$ is a vector subspace [respectively, closed vector subspace] of $\mathcal{H}$, by ri $C$ [respectively, sri $C$ ]. See [8, 46] for background on convex analysis and monotone operators.

\section{General algorithm}

We start with three preliminary results. The first one is an error-tolerant version of a forwardbackward-forward splitting algorithm originally proposed by Tseng [43, Theorem 3.4(b)].

Lemma 2.1 [13, Theorem 2.5(i)-(ii)] Let $\mathcal{K}$ be a real Hilbert space, let $\boldsymbol{P}: \mathcal{K} \rightarrow 2^{\mathcal{K}}$ be maximally monotone, and let $\boldsymbol{Q}: \mathcal{K} \rightarrow \mathcal{K}$ be monotone and $\chi$-Lipschitzian for some $\chi \in] 0,+\infty[$. Suppose that $\operatorname{zer}(\boldsymbol{P}+\boldsymbol{Q}) \neq \varnothing$. Let $\left(\boldsymbol{a}_{n}\right)_{n \in \mathbb{N}},\left(\boldsymbol{b}_{n}\right)_{n \in \mathbb{N}}$, and $\left(\boldsymbol{c}_{n}\right)_{n \in \mathbb{N}}$ be absolutely summable sequences in $\mathcal{K}$, let $\boldsymbol{w}_{0} \in \mathcal{K}$, let $\left.\varepsilon \in\right] 0,1 /(\chi+1)\left[\right.$, let $\left(\gamma_{n}\right)_{n \in \mathbb{N}}$ be a sequence in $[\varepsilon,(1-\varepsilon) / \chi]$, and set

$$
\begin{aligned}
& \text { For } n=0,1, \ldots \\
& \qquad \begin{array}{l}
\boldsymbol{s}_{n}=\boldsymbol{w}_{n}-\gamma_{n}\left(\boldsymbol{Q} \boldsymbol{w}_{n}+\boldsymbol{a}_{n}\right) \\
\boldsymbol{p}_{n}=J_{\gamma_{n} \boldsymbol{P}} \boldsymbol{s}_{n}+\boldsymbol{b}_{n} \\
\boldsymbol{q}_{n}=\boldsymbol{p}_{n}-\gamma_{n}\left(\boldsymbol{Q} \boldsymbol{p}_{n}+\boldsymbol{c}_{n}\right) \\
\boldsymbol{w}_{n+1}=\boldsymbol{w}_{n}-\boldsymbol{s}_{n}+\boldsymbol{q}_{n} .
\end{array}
\end{aligned}
$$

Then $\sum_{n \in \mathbb{N}}\left\|\boldsymbol{w}_{n}-\boldsymbol{p}_{n}\right\|^{2}<+\infty$ and there exists $\overline{\boldsymbol{w}} \in \operatorname{zer}(\boldsymbol{P}+\boldsymbol{Q})$ such that $\boldsymbol{w}_{n} \rightarrow \overline{\boldsymbol{w}}$ and $\boldsymbol{p}_{n} \rightarrow \overline{\boldsymbol{w}}$.

Lemma 2.2 [8, Proposition 23.15(ii) and 23.18] Let $\mathcal{H}$ be a real Hilbert space, let $A: \mathcal{H} \rightarrow 2^{\mathcal{H}}$ be a maximally monotone operator, let $\gamma \in] 0,+\infty\left[\right.$, and let $x$ and $r$ be in $\mathcal{H}$. Then $J_{\gamma\left(r+A^{-1}\right)} x=$ $x-\gamma\left(r+J_{\gamma^{-1} A}\left(\gamma^{-1} x-r\right)\right)$.

Lemma 2.3 [13, Proposition 2.8] Let $\mathcal{H}$ and $\mathcal{G}$ be two real Hilbert spaces, let $\boldsymbol{E}: \mathcal{H} \rightarrow 2^{\mathcal{H}}$ and $\boldsymbol{F}: \mathcal{G} \rightarrow 2^{\mathcal{G}}$ be maximally monotone, let $\boldsymbol{L} \in \mathcal{B}(\mathcal{H}, \mathcal{G})$, let $\boldsymbol{z} \in \mathcal{H}$, and let $\boldsymbol{r} \in \mathcal{G}$. Set $\mathcal{K}=\mathcal{H} \oplus \mathcal{G}$,

$$
\left\{\begin{array}{l}
\boldsymbol{M}: \mathcal{K} \rightarrow 2^{\mathcal{K}}:(\boldsymbol{x}, \boldsymbol{v}) \mapsto(-\boldsymbol{z}+\boldsymbol{E} \boldsymbol{x}) \times\left(\boldsymbol{r}+\boldsymbol{F}^{-1} \boldsymbol{v}\right) \\
\boldsymbol{S}: \mathcal{K} \rightarrow \mathcal{K}:(\boldsymbol{x}, \boldsymbol{v}) \mapsto\left(\boldsymbol{L}^{*} \boldsymbol{v},-\boldsymbol{L x}\right)
\end{array}\right.
$$


and

$$
\left\{\begin{array}{l}
\mathfrak{P}=\left\{\boldsymbol{x} \in \mathcal{H} \mid \boldsymbol{z} \in \boldsymbol{E} \boldsymbol{x}+\boldsymbol{L}^{*}(\boldsymbol{F}(\boldsymbol{L} \boldsymbol{x}-\boldsymbol{r}))\right\} \\
\mathfrak{D}=\left\{\boldsymbol{v} \in \mathcal{G} \mid-\boldsymbol{r} \in-\boldsymbol{L}\left(\boldsymbol{E}^{-1}\left(\boldsymbol{z}-\boldsymbol{L}^{*} \boldsymbol{v}\right)\right)+\boldsymbol{F}^{-1} \boldsymbol{v}\right\}
\end{array}\right.
$$

Then $\operatorname{zer}(\boldsymbol{M}+\boldsymbol{S})$ is a closed convex subset of $\mathfrak{P} \times \mathfrak{D}$, and $\mathfrak{P} \neq \varnothing \Leftrightarrow \operatorname{zer}(\boldsymbol{M}+\boldsymbol{S}) \neq \varnothing \Leftrightarrow \mathfrak{D} \neq \varnothing$.

The following theorem contains our algorithm for solving Problem 1.1 and states its main asymptotic properties. In this primal-dual splitting algorithm, each single-valued operators is used explicitly, while each set-valued operators is activated via its resolvent. Approximations in the evaluations of the operators are tolerated and modeled by absolutely summable error sequences. The algorithm consists of three main loops, each of which can be implemented on a parallel architecture.

Theorem 2.4 Consider the setting of Problem 1.1 For every $i \in\{1, \ldots, m\}$, let $\left(a_{1, i, n}\right)_{n \in \mathbb{N}},\left(b_{1, i, n}\right)_{n \in \mathbb{N}}$, and $\left(c_{1, i, n}\right)_{n \in \mathbb{N}}$ be absolutely summable sequences in $\mathcal{H}_{i}$ and, for every $k \in\{1, \ldots, K\}$, let $\left(a_{2, k, n}\right)_{n \in \mathbb{N}}$, $\left(b_{2, k, n}\right)_{n \in \mathbb{N}}$, and $\left(c_{2, k, n}\right)_{n \in \mathbb{N}}$ be absolutely summable sequences in $\mathcal{G}_{k}$. Let $x_{1,0} \in \mathcal{H}_{1}, \ldots, x_{m, 0} \in \mathcal{H}_{m}$, $v_{1,0} \in \mathcal{G}_{1}, \ldots, v_{K, 0} \in \mathcal{G}_{K}$, let $\left.\varepsilon \in\right] 0,1 /(\beta+1)\left[\right.$, let $\left(\gamma_{n}\right)_{n \in \mathbb{N}}$ be a sequence in $[\varepsilon,(1-\varepsilon) / \beta]$, and set

$$
\begin{aligned}
& \text { For } n=0,1, \ldots \\
& \text { For } i=1, \ldots, m \\
& \left\lfloor\begin{array}{l}
s_{1, i, n}=x_{i, n}-\gamma_{n}\left(C_{i} x_{i, n}+\sum_{k=1}^{K} L_{k i}^{*} v_{k, n}+a_{1, i, n}\right) \\
p_{1, i, n}=J_{\gamma_{n} A_{i}}\left(s_{1, i, n}+\gamma_{n} z_{i}\right)+b_{1, i, n}
\end{array}\right. \\
& \text { For } k=1, \ldots, K \\
& \mid \begin{array}{l}
s_{2, k, n}=v_{k, n}-\gamma_{n}\left(D_{k}^{-1} v_{k, n}-\sum_{i=1}^{m} L_{k i} x_{i, n}+a_{2, k, n}\right) \\
p_{2, k, n}=s_{2, k, n}-\gamma_{n}\left(r_{k}+J_{\gamma_{n}^{-1} B_{k}}\left(\gamma_{n}^{-1} s_{2, k, n}-r_{k}\right)+b_{2, k, n}\right)
\end{array} \\
& q_{2, k, n}=p_{2, k, n}-\gamma_{n}\left(D_{k}^{-1} p_{2, k, n}-\sum_{i=1}^{m} L_{k i} p_{1, i, n}+c_{2, k, n}\right) \\
& v_{k, n+1}=v_{k, n}-s_{2, k, n}+q_{2, k, n} \\
& \text { For } i=1, \ldots, m \\
& \left\lfloor\begin{array}{l}
q_{1, i, n}=p_{1, i, n}-\gamma_{n}\left(C_{i} p_{1, i, n}+\sum_{k=1}^{K} L_{k i}^{*} p_{2, k, n}+c_{1, i, n}\right) \\
x_{i, n+1}=x_{i, n}-s_{1, i, n}+q_{1, i, n}
\end{array}\right.
\end{aligned}
$$

Then the following hold.

(i) $(\forall i \in\{1, \ldots, m\}) \sum_{n \in \mathbb{N}}\left\|x_{i, n}-p_{1, i, n}\right\|^{2}<+\infty$.

(ii) $(\forall k \in\{1, \ldots, K\}) \sum_{n \in \mathbb{N}}\left\|v_{k, n}-p_{2, k, n}\right\|^{2}<+\infty$.

(iii) There exist a solution $\left(\overline{x_{1}}, \ldots, \overline{x_{m}}\right)$ to (1.2) and a solution $\left(\overline{v_{1}}, \ldots, \overline{v_{K}}\right)$ to (1.3) such that the following hold.

(a) $(\forall i \in\{1, \ldots, m\}) z_{i}-\sum_{k=1}^{K} L_{k i}^{*} \overline{v_{k}} \in A_{i} \overline{x_{i}}+C_{i} \overline{x_{i}}$.

(b) $(\forall k \in\{1, \ldots, K\}) \sum_{i=1}^{m} L_{k i} \overline{x_{i}}-r_{k} \in B_{k}^{-1} \overline{v_{k}}+D_{k}^{-1} \overline{v_{k}}$.

(c) $(\forall i \in\{1, \ldots, m\}) x_{i, n} \rightarrow \overline{x_{i}}$ and $p_{1, i, n} \rightarrow \overline{x_{i}}$.

(d) $(\forall k \in\{1, \ldots, K\}) v_{k, n} \rightarrow \overline{v_{k}}$ and $p_{2, k, n} \rightarrow \overline{v_{k}}$.

(e) Suppose that, for some $j \in\{1, \ldots, m\}, A_{j}$ or $C_{j}$ is uniformly monotone at $\overline{x_{j}}$. Then $x_{j, n} \rightarrow$ $\overline{x_{j}}$ and $p_{1, j, n} \rightarrow \overline{x_{j}}$. 
(f) Suppose that, for some $l \in\{1, \ldots, K\}, B_{l}$ or $D_{l}$ is couniformly monotone at $\overline{v_{l}}$. Then $v_{l, n} \rightarrow \overline{v_{l}}$ and $p_{2, l, n} \rightarrow \bar{v}_{l}$.

Proof. Let us introduce the Hilbert direct sums

$$
\mathcal{H}=\mathcal{H}_{1} \oplus \cdots \oplus \mathcal{H}_{m}, \quad \mathcal{G}=\mathcal{G}_{1} \oplus \cdots \oplus \mathcal{G}_{K}, \quad \text { and } \quad \mathcal{K}=\mathcal{H} \oplus \mathcal{G}
$$

and let us denote by $\boldsymbol{x}=\left(x_{i}\right)_{1 \leqslant i \leqslant m}$ and $\boldsymbol{v}=\left(v_{k}\right)_{1 \leqslant k \leqslant K}$ generic elements in $\mathcal{H}$ and $\mathcal{G}$, respectively. We also define

$$
\left\{\begin{array} { l } 
{ \boldsymbol { A } : \mathcal { H } \rightarrow 2 ^ { \mathcal { H } } : \boldsymbol { x } \mapsto \underset { i = 1 } { \times } A _ { i } x _ { i } } \\
{ \boldsymbol { C } : \mathcal { H } \rightarrow \mathcal { H } : \boldsymbol { x } \mapsto ( C _ { i } x _ { i } ) _ { 1 \leqslant i \leqslant m } } \\
{ \boldsymbol { E } = \boldsymbol { A } + \boldsymbol { C } } \\
{ \boldsymbol { L } : \mathcal { H } \rightarrow \mathcal { G } : \boldsymbol { x } \mapsto ( \sum _ { i = 1 } ^ { m } L _ { k i } x _ { i } ) _ { 1 \leqslant k \leqslant K } } \\
{ \boldsymbol { z } = ( z _ { i } ) _ { 1 \leqslant i \leqslant m } }
\end{array} \quad \text { and } \quad \left\{\begin{array}{l}
\boldsymbol{B}: \mathcal{G} \rightarrow 2^{\mathcal{G}}: \boldsymbol{v} \mapsto \underset{k=1}{\times} B_{k} v_{k} \\
\boldsymbol{D}: \mathcal{G} \rightarrow 2^{\mathcal{G}}: \boldsymbol{v} \mapsto \underset{k=1}{\times} D_{k} v_{k} \\
\boldsymbol{F}=\boldsymbol{B} \square \boldsymbol{D} \\
\boldsymbol{r}=\left(r_{k}\right)_{1 \leqslant k \leqslant K} .
\end{array}\right.\right.
$$

It follows from [8, Proposition 20.22 and 20.23, Corollaries 20.25 and 24.4(i)] that $\boldsymbol{A}, \boldsymbol{B}, \boldsymbol{C}, \boldsymbol{D}, \boldsymbol{E}$, and $\boldsymbol{F}$ are maximally monotone. Moreover, $\boldsymbol{L} \in \mathcal{B}(\mathcal{H}, \mathcal{G}), \boldsymbol{L}^{*}: \mathcal{G} \rightarrow \mathcal{H}: \boldsymbol{v} \mapsto\left(\sum_{k=1}^{K} L_{k i}^{*} v_{k}\right)_{1 \leqslant i \leqslant m}$, and

$$
(\forall \boldsymbol{x} \in \mathcal{H}) \quad\|\boldsymbol{L} \boldsymbol{x}\|^{2}=\sum_{k=1}^{K}\left\|\sum_{i=1}^{m} L_{k i} x_{i}\right\|^{2} \leqslant \lambda\|\boldsymbol{x}\|^{2} .
$$

Next, we set

$$
\left\{\begin{array}{l}
\boldsymbol{M}: \mathcal{K} \rightarrow 2^{\mathcal{K}}:(\boldsymbol{x}, \boldsymbol{v}) \mapsto(-\boldsymbol{z}+\boldsymbol{E} \boldsymbol{x}) \times\left(\boldsymbol{r}+\boldsymbol{F}^{-1} \boldsymbol{v}\right) \\
\boldsymbol{P}: \mathcal{K} \rightarrow 2^{\mathcal{K}}:(\boldsymbol{x}, \boldsymbol{v}) \mapsto(-\boldsymbol{z}+\boldsymbol{A x}) \times\left(\boldsymbol{r}+\boldsymbol{B}^{-1} \boldsymbol{v}\right) \\
\boldsymbol{Q}: \mathcal{K} \rightarrow \mathcal{K}:(\boldsymbol{x}, \boldsymbol{v}) \mapsto\left(\boldsymbol{C} \boldsymbol{x}+\boldsymbol{L}^{*} \boldsymbol{v}, \boldsymbol{D}^{-1} \boldsymbol{v}-\boldsymbol{L} \boldsymbol{x}\right) \\
\boldsymbol{R}: \mathcal{K} \rightarrow \mathcal{K}:(\boldsymbol{x}, \boldsymbol{v}) \mapsto\left(\boldsymbol{C x}, \boldsymbol{D}^{-1} \boldsymbol{v}\right) \\
\boldsymbol{S}: \mathcal{K} \rightarrow \mathcal{K}:(\boldsymbol{x}, \boldsymbol{v}) \mapsto\left(\boldsymbol{L}^{*} \boldsymbol{v},-\boldsymbol{L x}\right) .
\end{array}\right.
$$

Note that

$$
\operatorname{zer}(\boldsymbol{P}+\boldsymbol{Q})=\left\{(\boldsymbol{x}, \boldsymbol{v}) \in \mathcal{H} \oplus \mathcal{G} \mid \boldsymbol{z}-\boldsymbol{L}^{*} \boldsymbol{v} \in \boldsymbol{A} \boldsymbol{x}+\boldsymbol{C} \boldsymbol{x} \quad \text { and } \quad \boldsymbol{L} \boldsymbol{x}-\boldsymbol{r} \in \boldsymbol{B}^{-1} \boldsymbol{v}+\boldsymbol{D}^{-1} \boldsymbol{v}\right\} .
$$

Furthermore, in view of [8, Propositions 20.22 and 20.23], $\boldsymbol{P}$ is maximally monotone, and Lemma 2.2 and [8, Proposition 23.16] yield

$$
\begin{gathered}
(\forall \gamma \in] 0,+\infty[)(\forall \boldsymbol{x} \in \mathcal{H})(\forall \boldsymbol{v} \in \mathcal{G}) \quad J_{\gamma \boldsymbol{P}}(\boldsymbol{x}, \boldsymbol{v})=\left(J_{\gamma A_{1}}\left(x_{1}+\gamma z_{1}\right), \ldots, J_{\gamma A_{m}}\left(x_{m}+\gamma z_{m}\right),\right. \\
\left.v_{1}-\gamma\left(r_{1}+J_{\gamma^{-1} B_{1}}\left(\gamma^{-1} v_{1}-r_{1}\right)\right), \ldots, v_{K}-\gamma\left(r_{K}+J_{\gamma^{-1} B_{K}}\left(\gamma^{-1} v_{K}-r_{K}\right)\right)\right) .
\end{gathered}
$$

On the other hand, since $\boldsymbol{C}$ and $\boldsymbol{D}^{-1}$ are monotone and Lipschitzian with, respectively, constants $\mu=\max _{1 \leqslant i \leqslant m} \mu_{i}$ and $\nu=\max _{1 \leqslant k \leqslant K} \nu_{k}, \boldsymbol{R}$ is monotone and Lipschitzian with constant $\max \{\mu, \nu\}$. In addition, it follows from [13, Proposition 2.7(ii)] and (2.7) that $S \in \mathcal{B}(\mathcal{K}, \mathcal{K})$ is a skew (hence monotone) operator with $\|\boldsymbol{S}\|=\|\boldsymbol{L}\| \leqslant \sqrt{\lambda}$. Altogether, since $\boldsymbol{Q}=\boldsymbol{R}+\boldsymbol{S}$, we derive from (1.1) that

$\boldsymbol{P}$ is maximally monotone and $\boldsymbol{Q}$ is monotone and $\beta$-Lipschitzian. 
Let us call $\mathfrak{P}$ and $\mathfrak{D}$ the sets of solutions to (1.2) and (1.3), respectively. It follows from (2.6) that

$$
\left\{\begin{array}{l}
\mathfrak{P}=\left\{\boldsymbol{x} \in \mathcal{H} \mid \boldsymbol{z} \in \boldsymbol{E} \boldsymbol{x}+\boldsymbol{L}^{*}(\boldsymbol{F}(\boldsymbol{L} \boldsymbol{x}-\boldsymbol{r}))\right\} \\
\mathfrak{D}=\left\{\boldsymbol{v} \in \mathcal{G} \mid-\boldsymbol{r} \in-\boldsymbol{L}\left(\boldsymbol{E}^{-1}\left(\boldsymbol{z}-\boldsymbol{L}^{*} \boldsymbol{v}\right)\right)+\boldsymbol{F}^{-1} \boldsymbol{v}\right\} .
\end{array}\right.
$$

Hence, since $\mathfrak{P} \neq \varnothing$ by assumption, we deduce from Lemma 2.3 that

$$
\varnothing \neq \operatorname{zer}(\boldsymbol{M}+\boldsymbol{S})=\operatorname{zer}(\boldsymbol{P}+\boldsymbol{Q}) \subset \mathfrak{P} \times \mathfrak{D} .
$$

Thus, to solve Problem 1.1, it is enough to find a zero of $\boldsymbol{P}+\boldsymbol{Q}$. For every $n \in \mathbb{N}$, let us set

$$
\left\{\begin{array}{l}
\boldsymbol{w}_{n}=\left(x_{1, n}, \ldots, x_{m, n}, v_{1, n}, \ldots, v_{K, n}\right) \\
\boldsymbol{s}_{n}=\left(s_{1,1, n}, \ldots, s_{1, m, n}, s_{2,1, n}, \ldots, s_{2, K, n}\right) \\
\boldsymbol{p}_{n}=\left(p_{1,1, n}, \ldots, p_{1, m, n}, p_{2,1, n}, \ldots, p_{2, K, n}\right) \\
\boldsymbol{q}_{n}=\left(q_{1,1, n}, \ldots, q_{1, m, n}, q_{2,1, n}, \ldots, q_{2, K, n}\right)
\end{array}\right.
$$

and

$$
\left\{\begin{array}{l}
\boldsymbol{a}_{n}=\left(a_{1,1, n}, \ldots, a_{1, m, n}, a_{2,1, n}, \ldots, a_{2, K, n}\right) \\
\boldsymbol{b}_{n}=\left(b_{1,1, n}, \ldots, b_{1, m, n},-\gamma_{n} b_{2,1, n}, \ldots,-\gamma_{n} b_{2, K, n}\right) \\
\boldsymbol{c}_{n}=\left(c_{1,1, n}, \ldots, c_{1, m, n}, c_{2,1, n}, \ldots, c_{2, K, n}\right)
\end{array}\right.
$$

Then, using (2.6), (2.8), and (2.10), we see that (2.4) reduces to (2.1). Moreover, our assumptions and (2.5) imply that $\left(\boldsymbol{a}_{n}\right)_{n \in \mathbb{N}},\left(\boldsymbol{b}_{n}\right)_{n \in \mathbb{N}}$, and $\left(\boldsymbol{c}_{n}\right)_{n \in \mathbb{N}}$ are absolutely summable sequences in $\mathcal{K}$. Hence, it follows from (2.11), (2.13), and Lemma 2.1 that $\sum_{n \in \mathbb{N}}\left\|\boldsymbol{w}_{n}-\boldsymbol{p}_{n}\right\|^{2}<+\infty$ and that there exists $\overline{\boldsymbol{w}} \in \operatorname{zer}(\boldsymbol{P}+\boldsymbol{Q})$ such that $\boldsymbol{w}_{n} \rightarrow \overline{\boldsymbol{w}}$ and $\boldsymbol{p}_{n} \rightarrow \overline{\boldsymbol{w}}$. Upon setting $\overline{\boldsymbol{w}}=\left(\overline{x_{1}}, \ldots, \overline{x_{m}}, \overline{v_{1}}, \ldots, \overline{v_{K}}\right)$ and appealing to (2.5) and (2.9), we thus obtain assertions (i), (ii), and (iii)(a) (iii)(d),

(iii)(e); Let us introduce the variables

$$
(\forall i \in\{1, \ldots, m\})(\forall n \in \mathbb{N})\left\{\begin{array}{l}
\widetilde{s}_{1, i, n}=x_{i, n}-\gamma_{n}\left(C_{i} x_{i, n}+\sum_{k=1}^{K} L_{k i}^{*} v_{k, n}\right) \\
\widetilde{p}_{1, i, n}=J_{\gamma_{n} A_{i}}\left(\widetilde{s}_{1, i, n}+\gamma_{n} z_{i}\right)
\end{array}\right.
$$

and

$$
(\forall k \in\{1, \ldots, K\})(\forall n \in \mathbb{N})\left\{\begin{array}{l}
\widetilde{s}_{2, k, n}=v_{k, n}-\gamma_{n}\left(D_{k}^{-1} v_{k, n}-\sum_{i=1}^{m} L_{k i} x_{i, n}\right) \\
\widetilde{p}_{2, k, n}=\widetilde{s}_{2, k, n}-\gamma_{n}\left(r_{k}+J_{\gamma_{n}^{-1} B_{k}}\left(\gamma_{n}^{-1} \widetilde{s}_{2, k, n}-r_{k}\right)\right) .
\end{array}\right.
$$

It follows from (2.4) that

$$
(\forall i \in\{1, \ldots, m\})(\forall n \in \mathbb{N}) \quad\left\|s_{1, i, n}-\widetilde{s}_{1, i, n}\right\|=\gamma_{n}\left\|a_{1, i, n}\right\| \leqslant \beta^{-1}\left\|a_{1, i, n}\right\| .
$$

Hence, by virtue of the nonexpansiveness of the resolvents [8, Proposition 23.7], we have

$$
\begin{aligned}
(\forall i \in\{1, \ldots, m\})(\forall n \in \mathbb{N})\left\|p_{1, i, n}-\widetilde{p}_{1, i, n}\right\| & =\left\|J_{\gamma_{n} A_{i}}\left(s_{1, i, n}+\gamma_{n} z_{i}\right)+b_{1, i, n}-J_{\gamma_{n} A_{i}}\left(\widetilde{s}_{1, i, n}+\gamma_{n} z_{i}\right)\right\| \\
& \leqslant\left\|s_{1, i, n}-\widetilde{s}_{1, i, n}\right\|+\left\|b_{1, i, n}\right\| \\
& \leqslant \beta^{-1}\left\|a_{1, i, n}\right\|+\left\|b_{1, i, n}\right\| .
\end{aligned}
$$


In turn, since, for every $i \in\{1, \ldots, m\},\left(a_{1, i, n}\right)_{n \in \mathbb{N}}$ and $\left(b_{1, i, n}\right)_{n \in \mathbb{N}}$ are absolutely summable, we get

$$
(\forall i \in\{1, \ldots, m\}) \quad s_{1, i, n}-\widetilde{s}_{1, i, n} \rightarrow 0 \quad \text { and } \quad p_{1, i, n}-\widetilde{p}_{1, i, n} \rightarrow 0 .
$$

Likewise, we derive from (2.4) and (2.17) that

$$
(\forall k \in\{1, \ldots, K\}) \quad s_{2, k, n}-\widetilde{s}_{2, k, n} \rightarrow 0 \quad \text { and } \quad p_{2, k, n}-\widetilde{p}_{2, k, n} \rightarrow 0 .
$$

On the other hand, we deduce from (iii)(a) that

$$
(\forall i \in\{1, \ldots, m\})\left(\exists u_{i} \in \mathcal{H}_{i}\right) \quad u_{i} \in A_{i} \overline{x_{i}} \quad \text { and } \quad z_{i}=u_{i}+\sum_{k=1}^{K} L_{k i}^{*} \overline{v_{k}}+C_{i} \overline{x_{i}},
$$

and from (iii)(b) that

$$
(\forall k \in\{1, \ldots, K\}) \quad \overline{v_{k}} \in B_{k}\left(\sum_{i=1}^{m} L_{k i} \overline{x_{i}}-r_{k}-D_{k}^{-1} \overline{v_{k}}\right) .
$$

In addition, (2.16) yields

$$
(\forall i \in\{1, \ldots, m\})(\forall n \in \mathbb{N}) \quad \frac{x_{i, n}-\widetilde{p}_{1, i, n}}{\gamma_{n}}-\sum_{k=1}^{K} L_{k i}^{*} v_{k, n}-C_{i} x_{i, n}+z_{i} \in A_{i} \widetilde{p}_{1, i, n},
$$

while (2.17) yields

$$
(\forall k \in\{1, \ldots, K\})(\forall n \in \mathbb{N}) \quad \widetilde{p}_{2, k, n} \in B_{k}\left(\frac{v_{k, n}-\widetilde{p}_{2, k, n}}{\gamma_{n}}+\sum_{i=1}^{m} L_{k i} x_{i, n}-r_{k}-D_{k}^{-1} v_{k, n}\right)
$$

Now, let us set

$$
\begin{array}{r}
(\forall n \in \mathbb{N}) \quad \delta_{n}=\sum_{k=1}^{K}\left(\frac{1}{\varepsilon}+\nu_{k}\right)\left\|v_{k, n}-\widetilde{p}_{2, k, n}\right\|\left\|\widetilde{p}_{2, k, n}-\overline{v_{k}}\right\| \quad \text { and } \quad(\forall i \in\{1, \ldots, m\}) \\
\alpha_{i, n}=\left\|\widetilde{p}_{1, i, n}-x_{i, n}\right\|\left(\frac{1}{\varepsilon}\left\|\widetilde{p}_{1, i, n}-\overline{x_{i}}\right\|+\mu_{i}\left\|x_{i, n}-\overline{x_{i}}\right\|+\sum_{k=1}^{K}\left\|L_{k i}\right\|\left\|v_{k, n}-\overline{v_{k}}\right\|\right)
\end{array}
$$

It follows from (i), (ii), (iii)(c), (iii)(d), (2.20), and (2.21) that

$$
\delta_{n} \rightarrow 0 \quad \text { and } \quad(\forall i \in\{1, \ldots, m\}) \quad \alpha_{i, n} \rightarrow 0 .
$$

Using the Cauchy-Schwarz inequality, the Lipschitz-continuity and the monotonicity of the operators $\left(C_{i}\right)_{1 \leqslant i \leqslant m}$, (2.22), (2.24), and the monotonicity of the operators $\left(A_{i}\right)_{1 \leqslant i \leqslant m}$, we obtain

$$
(\forall i \in\{1, \ldots, m\})(\forall n \in \mathbb{N}) \quad \alpha_{i, n}+\left\langle x_{i, n}-\overline{x_{i}} \mid \sum_{k=1}^{K} L_{k i}^{*}\left(\overline{v_{k}}-v_{k, n}\right)\right\rangle
$$




$$
\begin{aligned}
\geqslant & \left\|\widetilde{p}_{1, i, n}-x_{i, n}\right\|\left(\varepsilon^{-1}\left\|\widetilde{p}_{1, i, n}-\overline{x_{i}}\right\|+\left\|C_{i} x_{i, n}-C_{i} \overline{x_{i}}\right\|\right)+\left\langle\widetilde{p}_{1, i, n}-x_{i, n} \mid \sum_{k=1}^{K} L_{k i}^{*}\left(\overline{v_{k}}-v_{k, n}\right)\right\rangle \\
& +\left\langle x_{i, n}-\overline{x_{i}} \mid \sum_{k=1}^{K} L_{k i}^{*}\left(\overline{v_{k}}-v_{k, n}\right)\right\rangle \\
= & \left\|\widetilde{p}_{1, i, n}-x_{i, n}\right\|\left(\varepsilon^{-1}\left\|\widetilde{p}_{1, i, n}-\overline{x_{i}}\right\|+\left\|C_{i} x_{i, n}-C_{i} \overline{x_{i}}\right\|\right)+\left\langle\widetilde{p}_{1, i, n}-\overline{x_{i}} \mid \sum_{k=1}^{K} L_{k i}^{*}\left(\overline{v_{k}}-v_{k, n}\right)\right\rangle \\
\geqslant & \left\langle\widetilde{p}_{1, i, n}-\overline{x_{i}} \mid \frac{x_{i, n}-\widetilde{p}_{1, i, n}}{\gamma_{n}}+\sum_{k=1}^{K} L_{k i}^{*}\left(\overline{v_{k}}-v_{k, n}\right)\right\rangle+\left\langle\widetilde{p}_{1, i, n}-x_{i, n} \mid C_{i} \overline{x_{i}}-C_{i} x_{i, n}\right\rangle \\
= & \left\langle\widetilde{p}_{1, i, n}-\overline{x_{i}} \mid \frac{x_{i, n}-\widetilde{p}_{1, i, n}}{\gamma_{n}}-\sum_{k=1}^{K} L_{k i}^{*} v_{k, n}-C_{i} x_{i, n}+\sum_{k=1}^{K} L_{k i}^{*} \overline{v_{k}}+C_{i} \overline{x_{i}}\right\rangle \\
& +\left\langle x_{i, n}-\overline{x_{i}} \mid C_{i} x_{i, n}-C_{i} \overline{x_{i}}\right\rangle \\
= & \left\langle\widetilde{p}_{1, i, n}-\overline{x_{i}} \mid \frac{x_{i, n}-\widetilde{p}_{1, i, n}}{\gamma_{n}}-\sum_{k=1}^{K} L_{k i}^{*} v_{k, n}-C_{i} x_{i, n}+z_{i}-u_{i}\right\rangle \\
& +\left\langle x_{i, n}-\overline{x_{i}} \mid C_{i} x_{i, n}-C_{i} \overline{x_{i}}\right\rangle \\
\geqslant & \left\langle\widetilde{p}_{1, i, n}-\overline{x_{i}} \mid\left(\frac{x_{i, n}-\widetilde{p}_{1, i, n}}{\gamma_{n}}-\sum_{k=1}^{K} L_{k i}^{*} v_{k, n}-C_{i} x_{i, n}+z_{i}\right)-u_{i}\right\rangle \\
& 0
\end{aligned}
$$

On the other hand, since the operators $\left(D_{k}^{-1}\right)_{1 \leqslant k \leqslant K}$ are Lipschitzian and monotone, and since the operators $\left(B_{k}\right)_{1 \leqslant k \leqslant K}$ are monotone, we deduce from (2.26), (2.23), and (2.25) that

$$
\begin{aligned}
&(\forall l \in\{1 \ldots, K\})(\forall n \in \mathbb{N}) \quad \delta_{n}+\sum_{i=1}^{m}\left\langle x_{i, n}-\overline{x_{i}} \mid \sum_{k=1}^{K} L_{k i}^{*}\left(\widetilde{p}_{2, k, n}-\overline{v_{k}}\right)\right\rangle \\
& \geqslant \sum_{k=1}^{K}\left\langle\frac{v_{k, n}-\widetilde{p}_{2, k, n}}{\gamma_{n}}+D_{k}^{-1} \widetilde{p}_{2, k, n}-D_{k}^{-1} v_{k, n}+\sum_{i=1}^{m} L_{k i}\left(x_{i, n}-\overline{x_{i}}\right) \mid \widetilde{p}_{2, k, n}-\overline{v_{k}}\right\rangle \\
&= \sum_{k=1}^{K}\left\langle\left(\frac{v_{k, n}-\widetilde{p}_{2, k, n}}{\gamma_{n}}+\sum_{i=1}^{m} L_{k i} x_{i, n}-r_{k}-D_{k}^{-1} v_{k, n}\right)-\left(\sum_{i=1}^{m} L_{k i} \overline{x_{i}}-r_{k}-D_{k}^{-1} \overline{v_{k}}\right) \mid \widetilde{p}_{2, k, n}-\overline{v_{k}}\right\rangle \\
&+\sum_{k=1}^{K}\left\langle D_{k}^{-1} \widetilde{p}_{2, k, n}-D_{k}^{-1} \overline{v_{k}} \mid \widetilde{p}_{2, k, n}-\overline{v_{k}}\right\rangle \\
& \geqslant\left\langle\left(\frac{v_{l, n}-\widetilde{p}_{2, l, n}}{\gamma_{n}}+\sum_{i=1}^{m} L_{l i} x_{i, n}-r_{l}-D_{l}^{-1} v_{l, n}\right)-\left(\sum_{i=1}^{m} L_{l i} \overline{x_{i}}-r_{l}-D_{l}^{-1} \overline{v_{l}}\right) \mid \widetilde{p}_{2, l, n}-\overline{v_{l}}\right\rangle \\
&+\left\langle D_{l}^{-1} \widetilde{p}_{2, l, n}-D_{l}^{-1} \bar{v}_{l} \mid \widetilde{p}_{2, l, n}-\overline{v_{l}}\right\rangle \\
& \geqslant\left\langle\left(\frac{v_{l, n}-\widetilde{p}_{2, l, n}}{\gamma_{n}}+\sum_{i=1}^{m} L_{l i} x_{i, n}-r_{l}-D_{l}^{-1} v_{l, n}\right)-\left(\sum_{i=1}^{m} L_{l i} \overline{x_{i}}-r_{l}-D_{l}^{-1} \overline{v_{l}}\right) \mid \widetilde{p}_{2, l, n}-\overline{v_{l}}\right\rangle \\
& \geqslant 0 .
\end{aligned}
$$

We consider two cases. 
- If $A_{j}$ is uniformly monotone at $\overline{x_{j}}$, then, in view of (2.29), (2.22), (2.24), and (1.8), there exists an increasing function $\phi_{A_{j}}:[0,+\infty[\rightarrow[0,+\infty]$ that vanishes only at 0 such that

$$
(\forall n \in \mathbb{N}) \quad \alpha_{j, n}+\left\langle x_{j, n}-\overline{x_{j}} \mid \sum_{k=1}^{K} L_{k j}^{*}\left(\overline{v_{k}}-v_{k, n}\right)\right\rangle \geqslant \phi_{A_{j}}\left(\left\|\widetilde{p}_{1, j, n}-\overline{x_{j}}\right\|\right) .
$$

Combining (2.34), (2.30), and (2.35) yields

$$
(\forall n \in \mathbb{N}) \quad \delta_{n}+\sum_{i=1}^{m} \alpha_{i, n}+\sum_{i=1}^{m}\left\langle x_{i, n}-\overline{x_{i}} \mid \sum_{k=1}^{K} L_{k i}^{*}\left(\widetilde{p}_{2, k, n}-v_{k, n}\right)\right\rangle \geqslant \phi_{A_{j}}\left(\left\|\widetilde{p}_{1, j, n}-\overline{x_{j}}\right\|\right) .
$$

It follows from (2.27), (ii), (iii)(c), (2.21), and [8, Lemma 2.41(iii)] that $\phi_{A_{j}}\left(\left\|\widetilde{p}_{1, j, n}-\overline{x_{j}}\right\|\right) \rightarrow 0$ and, in turn, that $\widetilde{p}_{1, j, n} \rightarrow \overline{x_{j}}$. In view of (i)] and (2.20), we get $p_{1, j, n} \rightarrow \overline{x_{j}}$ and $x_{j, n} \rightarrow \overline{x_{j}}$.

- If $C_{j}$ is uniformly monotone at $\overline{x_{j}}$, then we derive from (2.34), (2.28), and (2.30) that there exists an increasing function $\phi_{C_{j}}:[0,+\infty[\rightarrow[0,+\infty]$ that vanishes only at 0 such that

$$
\begin{aligned}
(\forall n \in \mathbb{N}) \quad \delta_{n}+\sum_{i=1}^{m} \alpha_{i, n}+\sum_{i=1}^{m}\left\langle x_{i, n}-\overline{x_{i}} \mid \sum_{k=1}^{K} L_{k i}^{*}\left(\widetilde{p}_{2, k, n}-v_{k, n}\right)\right\rangle & \\
& \geqslant \phi_{C_{j}}\left(\left\|x_{j, n}-\overline{x_{j}}\right\|\right) .
\end{aligned}
$$

This implies that $\phi_{C_{j}}\left(\left\|x_{j, n}-\overline{x_{j}}\right\|\right) \rightarrow 0$ and hence that $x_{j, n} \rightarrow \overline{x_{j}}$. Finally, (i)]yields $p_{1, j, n} \rightarrow \overline{x_{j}}$.

(iii)(f): We consider two cases.

- If $B_{l}$ is couniformly monotone at $\overline{v_{l}}$, then (2.33), (2.23), and (2.25) imply that there exists an increasing function $\phi_{B_{l}^{-1}}:[0,+\infty[\rightarrow[0,+\infty]$ that vanishes only at 0 such that

$$
\begin{aligned}
& (\forall n \in \mathbb{N}) \quad \delta_{n}+\sum_{i=1}^{m}\left\langle x_{i, n}-\overline{x_{i}} \mid \sum_{k=1}^{K} L_{k i}^{*}\left(\widetilde{p}_{2, k, n}-\overline{v_{k}}\right)\right\rangle \\
& \geqslant\left\langle\left(\frac{v_{l, n}-\widetilde{p}_{2, l, n}}{\gamma_{n}}+\sum_{i=1}^{m} L_{l i} x_{i, n}-r_{l}-D_{l}^{-1} v_{l, n}\right)-\left(\sum_{i=1}^{m} L_{l i} \overline{x_{i}}-r_{l}-D_{l}^{-1} \overline{v_{l}}\right) \mid \widetilde{p}_{2, l, n}-\overline{v_{l}}\right\rangle \\
& \geqslant \phi_{B_{l}^{-1}}\left(\left\|\widetilde{p}_{2, l, n}-\overline{v_{l}}\right\|\right) .
\end{aligned}
$$

Combining this with (2.30) yields

$$
(\forall n \in \mathbb{N}) \quad \delta_{n}+\sum_{i=1}^{m} \alpha_{i, n}+\sum_{i=1}^{m}\left\langle x_{i, n}-\overline{x_{i}} \mid \sum_{k=1}^{K} L_{k i}^{*}\left(\widetilde{p}_{2, k, n}-v_{k, n}\right)\right\rangle \geqslant \phi_{B_{l}^{-1}}\left(\left\|\widetilde{p}_{2, l, n}-\bar{v}_{l}\right\|\right) .
$$

Hence, using (2.27), (ii), (iii)(c), (2.21), and [8, Lemma 2.41(iii)], we get $\phi_{B_{l}^{-1}}\left(\left\|\widetilde{p}_{2, l, n}-\bar{v}_{l}\right\|\right) \rightarrow$ 0 and, in turn, $\widetilde{p}_{2, l, n} \rightarrow \bar{v}_{l}$. Using to (2.21) and (ii), we conclude that $p_{2, l, n} \rightarrow \overline{v_{l}}$ and $v_{l, n} \rightarrow \overline{v_{l}}$.

- If $D_{l}$ is couniformly monotone at $\overline{v_{l}}$, then it follows from (2.32) and (2.34) that there exists an increasing function $\phi_{D_{l}^{-1}}:[0,+\infty[\rightarrow[0,+\infty]$ that vanishes only at 0 such that

$$
\begin{aligned}
(\forall n \in \mathbb{N}) \quad \delta_{n}+\sum_{i=1}^{m}\left\langle x_{i, n}-\overline{x_{i}} \mid \sum_{k=1}^{K} L_{k i}^{*}\left(\widetilde{p}_{2, k, n}-\overline{v_{k}}\right)\right\rangle & \geqslant\left\langle D_{l}^{-1} \widetilde{p}_{2, l, n}-D_{l}^{-1} \overline{v_{l}} \mid \widetilde{p}_{2, l, n}-\overline{v_{l}}\right\rangle \\
& \geqslant \phi_{D_{l}^{-1}}\left(\left\|\widetilde{p}_{2, l, n}-\overline{v_{l}}\right\|\right) .
\end{aligned}
$$


Thus, (2.30) yields

$$
(\forall n \in \mathbb{N}) \quad \delta_{n}+\sum_{i=1}^{m} \alpha_{i, n}+\sum_{i=1}^{m}\left\langle x_{i, n}-\overline{x_{i}} \mid \sum_{k=1}^{K} L_{k i}^{*}\left(\widetilde{p}_{2, k, n}-v_{k, n}\right)\right\rangle \geqslant \phi_{D_{l}^{-1}}\left(\left\|\widetilde{p}_{2, l, n}-\bar{v}_{l}\right\|\right),
$$

and we conclude as above.

Remark 2.5 When $m=1$, Theorem 2.4 specializes to [19, Theorem 3.1]. Our proof of Theorem 2.4)(i) (iii)(d) hinges on a self-contained application of Lemmas 2.3 and 2.1 in the primal-dual product space $\mathcal{K}$ of (2.5). Alternatively, these results could be obtained as an application of [19, Theorem 3.1] using the product space $\mathcal{H}$ of (2.5) as a primal space. This strategy, however, would not allow us to recover the strong convergence results of Theorem 2.4l(iii)(e).

Remark 2.6 It follows from the Cauchy-Schwarz inequality that, for every $\left(x_{i}\right)_{1 \leqslant i \leqslant m} \in \bigoplus_{i=1}^{m} \mathcal{H}_{i}$,

$$
\sum_{k=1}^{K}\left\|\sum_{i=1}^{m} L_{k i} x_{i}\right\|^{2} \leqslant \sum_{k=1}^{K}\left(\sum_{i=1}^{m}\left\|L_{k i}\right\|\left\|x_{i}\right\|\right)^{2} \leqslant \sum_{k=1}^{K}\left(\sum_{i=1}^{m}\left\|L_{k i}\right\|^{2}\right)\left(\sum_{i=1}^{m}\left\|x_{i}\right\|^{2}\right) .
$$

Hence, in general, one can use $\lambda=\sum_{k=1}^{K} \sum_{i=1}^{m}\left\|L_{k i}\right\|^{2}$ in (1.1). However, as will be seen in subsequent sections, this bound can be improved when the operator $\boldsymbol{L}$ of (2.6) has a special structure.

In the remainder the paper, we highlight a few instantiations of Theorem 2.4 that illustrate the variety of problems to which it can be applied and which are not explicitly solvable via existing techniques.

\section{Inclusions involving general parallel sums}

The first special case of Problem 1.1 we feature is an extension of a univariate inclusion problem investigated in [19], which involves parallel sums with monotone operators admitting Lipschitzian inverses. In the following formulation, we lift this restriction.

Problem 3.1 Let $\mathcal{H}$ be a real Hilbert space, let $K_{1}, K_{2}$, and $K$ be integers such that $0 \leqslant K_{1} \leqslant K_{2} \leqslant$ $K \geqslant 1$, let $z \in \mathcal{H}$, let $A: \mathcal{H} \rightarrow 2^{\mathcal{H}}$ be maximally monotone, and let $C: \mathcal{H} \rightarrow \mathcal{H}$ be monotone and $\mu$-Lipschitzian for some $\mu \in\left[0,+\infty\left[\right.\right.$. For every integer $k \in\{1, \ldots, K\}$, let $\mathcal{G}_{k}$ be a real Hilbert space, let $r_{k} \in \mathcal{G}_{k}$, let $B_{k}: \mathcal{G}_{k} \rightarrow 2^{\mathcal{G}_{k}}$ and $S_{k}: \mathcal{G}_{k} \rightarrow 2^{\mathcal{G}_{k}}$ be maximally monotone, and let $L_{k} \in \mathcal{B}\left(\mathcal{H}, \mathcal{G}_{k}\right)$; moreover, if $K_{1}+1 \leqslant k \leqslant K_{2}, S_{k}: \mathcal{G}_{k} \rightarrow \mathcal{G}_{k}$ is $\beta_{k}$-Lipschitzian for some $\beta_{k} \in[0,+\infty$ [, and, if $K_{2}+1 \leqslant k \leqslant K, S_{k}^{-1}: \mathcal{G}_{k} \rightarrow \mathcal{G}_{k}$ is $\beta_{k}$-Lipschitzian for some $\beta_{k} \in[0,+\infty[$. It is assumed that

$$
\beta=\max \left\{\mu, \beta_{K_{1}+1}, \ldots, \beta_{K}\right\}+\sqrt{1+\sum_{k=1}^{K}\left\|L_{k}\right\|^{2}}>0
$$

and that the inclusion

$$
\text { find } \bar{x} \in \mathcal{H} \text { such that } z \in A \bar{x}+\sum_{k=1}^{K} L_{k}^{*}\left(\left(B_{k} \square S_{k}\right)\left(L_{k} \bar{x}-r_{k}\right)\right)+C \bar{x}
$$


possesses at least one solution. Solve (3.2) together with the dual problem

find $\overline{v_{1}} \in \mathcal{G}_{1}, \ldots, \overline{v_{K}} \in \mathcal{G}_{K}$ such that

$$
(\forall k \in\{1, \ldots, K\}) \quad-r_{k} \in-L_{k}\left((A+C)^{-1}\left(z-\sum_{l=1}^{K} L_{l}^{*} \overline{v_{l}}\right)\right)+B_{k}^{-1} \overline{v_{k}}+S_{k}^{-1} \overline{v_{k}} .
$$

Proposition 3.2 Consider the setting of Problem 3.1. Let $\left(a_{1,1, n}\right)_{n \in \mathbb{N}},\left(b_{1,1, n}\right)_{n \in \mathbb{N}}$, and $\left(c_{1,1, n}\right)_{n \in \mathbb{N}}$ be absolutely summable sequences in $\mathcal{H}$. For every integer $k \in\{1, \ldots, K\}$, let $\left(a_{2, k, n}\right)_{n \in \mathbb{N}},\left(b_{2, k, n}\right)_{n \in \mathbb{N}}$, and $\left(c_{2, k, n}\right)_{n \in \mathbb{N}}$ be absolutely summable sequences in $\mathcal{G}_{k}$; moreover, if $1 \leqslant k \leqslant K_{1}$, let $\left(b_{1, k+1, n}\right)_{n \in \mathbb{N}}$ be an absolutely summable sequence in $\mathcal{G}_{k}$, and, if $K_{1}+1 \leqslant k \leqslant K_{2}$, let $\left(a_{1, k+1, n}\right)_{n \in \mathbb{N}}$ and $\left(c_{1, k+1, n}\right)_{n \in \mathbb{N}}$ be absolutely summable sequences in $\mathcal{G}_{k}$. Let $x_{0} \in \mathcal{H}, y_{1,0} \in \mathcal{G}_{1}, \ldots, y_{K_{2}, 0} \in \mathcal{G}_{K_{2}}, v_{1,0} \in \mathcal{G}_{1}, \ldots$, and $v_{K, 0} \in \mathcal{G}_{K}$, let $\left.\varepsilon \in\right] 0,1 /(\beta+1)\left[\right.$, let $\left(\gamma_{n}\right)_{n \in \mathbb{N}}$ be a sequence in $[\varepsilon,(1-\varepsilon) / \beta]$, and set

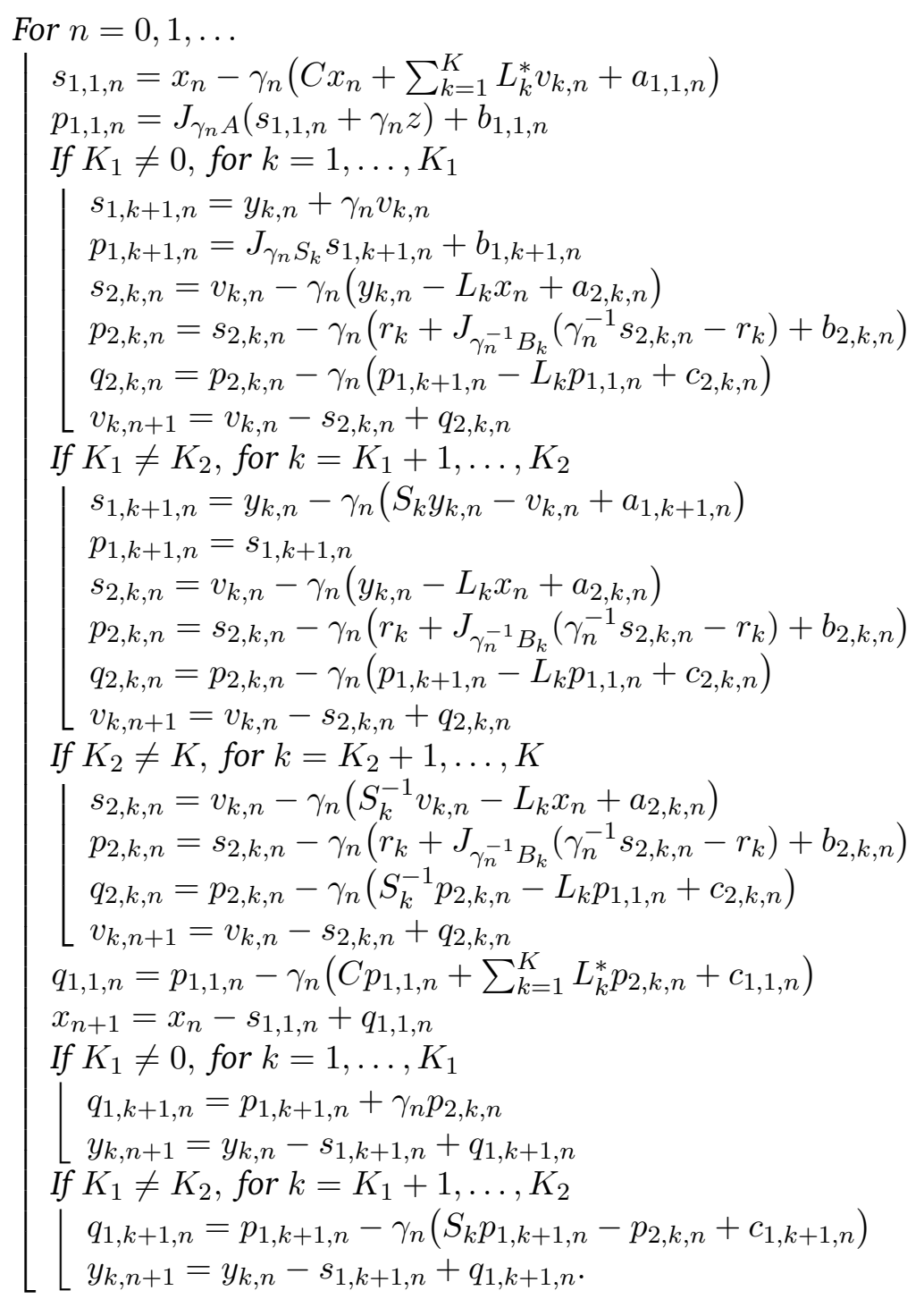

Then the following hold for some solution $\bar{x}$ to (3.2) and some solution $\left(\overline{v_{1}}, \ldots, \overline{v_{K}}\right)$ to (3.3).

(i) $x_{n} \rightarrow \bar{x}$ and $(\forall k \in\{1, \ldots, K\}) v_{k, n} \rightarrow \overline{v_{k}}$. 
(ii) Suppose that $A$ or $C$ is uniformly monotone at $\bar{x}$. Then $x_{n} \rightarrow \bar{x}$.

(iii) Suppose that, for some $l \in\{1, \ldots, K\}, B_{l}$ is couniformly monotone at $\overline{v_{l}}$. Then $v_{l, n} \rightarrow \overline{v_{l}}$.

(iv) Suppose that $K_{2} \neq K$ and that, for some $l \in\left\{K_{2}+1, \ldots, K\right\}, S_{l}$ is couniformly monotone at $\overline{v_{l}}$. Then $v_{l, n} \rightarrow \overline{v_{l}}$.

Proof. We assume that $K_{2} \neq 0$ and consider the auxiliary problem

find $\bar{x} \in \mathcal{H}, \overline{y_{1}} \in \mathcal{G}_{1}, \ldots, \overline{y_{K_{2}}} \in \mathcal{G}_{K_{2}}$ such that

$$
\left\{\begin{aligned}
z & \in A \bar{x}+\sum_{k=1}^{K_{2}} L_{k}^{*}\left(B_{k}\left(L_{k} \bar{x}-\overline{y_{k}}-r_{k}\right)\right)+\sum_{k=K_{2}+1}^{K} L_{k}^{*}\left(\left(B_{k} \square S_{k}\right)\left(L_{k} \bar{x}-r_{k}\right)\right)+C \bar{x} \\
0 & \in S_{1} \overline{y_{1}}-B_{1}\left(L_{1} \bar{x}-\overline{y_{1}}-r_{1}\right) \\
& \vdots \\
0 & \in S_{K_{2}} \overline{y_{K_{2}}}-B_{K_{2}}\left(L_{K_{2}} \bar{x}-\overline{y_{K_{2}}}-r_{K_{2}}\right)
\end{aligned}\right.
$$

together with the dual problem (3.3) (if $K_{2}=0$, (3.5) should be replaced by (3.2) and the resulting simplifications in the proof are straightforward). Let us show that solving the primal-dual problem (3.5) $/(3.3)$ is a special case of Problem 1.1 with

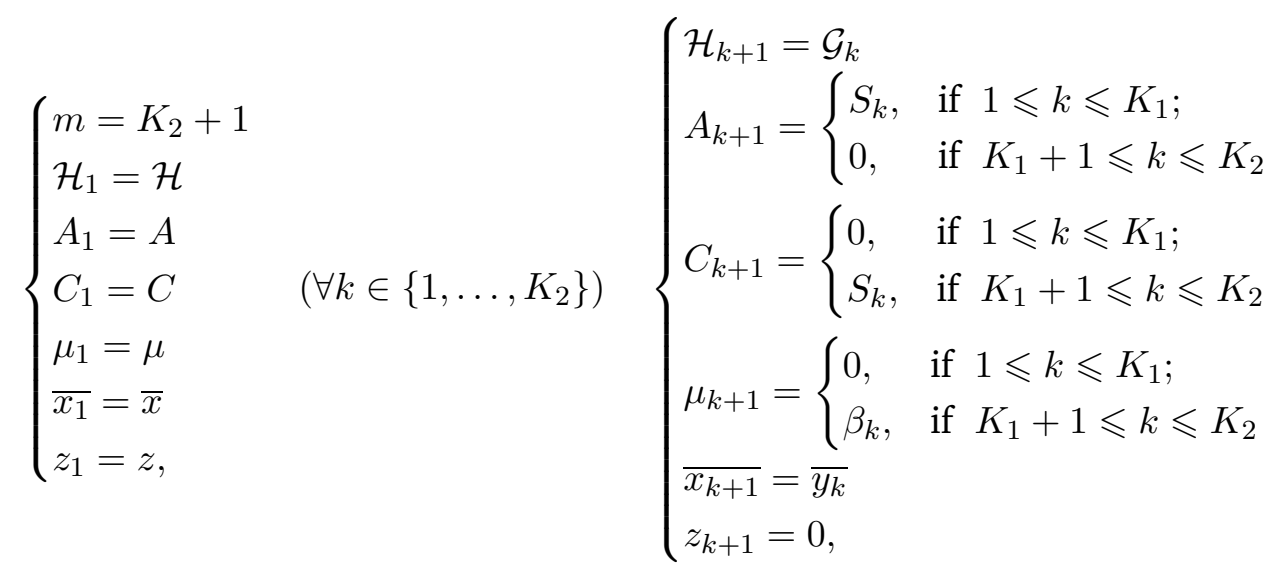

and

$$
(\forall k \in\{1, \ldots, K\})\left\{\begin{array}{l}
D_{k}= \begin{cases}\{0\}^{-1}, & \text { if } 1 \leqslant k \leqslant K_{2} \\
S_{k}, & \text { if } K_{2}+1 \leqslant k \leqslant K\end{cases} \\
\nu_{k+1}= \begin{cases}0, & \text { if } 1 \leqslant k \leqslant K_{2} \\
\beta_{k}, & \text { if } K_{2}+1 \leqslant k \leqslant K\end{cases} \\
L_{k 1}=L_{k} \\
\left(\forall i \in\left\{2, \ldots, K_{2}+1\right\}\right) L_{k i}= \begin{cases}-\mathrm{Id}, & \text { if } i=k+1 \\
0, & \text { otherwise. }\end{cases}
\end{array}\right.
$$

First, we note that, in this setting, (1.2) reduces to (3.5), and (1.3) to (3.3). Now define $\mathcal{H}$ and $\mathcal{G}$ as in (2.5), let $x \in \mathcal{H}$, let $\left(y_{k}\right)_{1 \leqslant k \leqslant K_{2}} \in \bigoplus_{k=1}^{K_{2}} \mathcal{G}_{k}$, set $\left(x_{i}\right)_{1 \leqslant i \leqslant m}=\left(x, y_{1}, \ldots, y_{K_{2}}\right) \in \mathcal{H}$, set $\boldsymbol{y}=\left(y_{1}, \ldots, y_{K_{2}}, 0, \ldots, 0\right) \in \mathcal{G}$, and set $\lambda=1+\sum_{k=1}^{K_{2}}\left\|L_{k}\right\|^{2}$. Then, using the Cauchy-Schwarz 
inequality in $\mathbb{R}^{2}$,

$$
\begin{aligned}
& \sum_{k=1}^{K}\left\|\sum_{i=1}^{m} L_{k i} x_{i}\right\|^{2}=\left\|\left(L_{k} x\right)_{1 \leqslant k \leqslant K_{2}}-\boldsymbol{y}\right\|^{2} \leqslant\left(\|\boldsymbol{y}\|+\left\|\left(L_{k} x\right)_{1 \leqslant k \leqslant K_{2}}\right\|\right)^{2} \\
& \leqslant\left(\|\boldsymbol{y}\|+\sqrt{\sum_{k=1}^{K_{2}}\left\|L_{k}\right\|^{2}}\|x\|\right)^{2} \leqslant\left(1+\sum_{k=1}^{K_{2}}\left\|L_{k}\right\|^{2}\right)\left(\|\boldsymbol{y}\|^{2}+\|x\|^{2}\right)=\lambda \sum_{i=1}^{m}\left\|x_{i}\right\|^{2} .
\end{aligned}
$$

Thus (1.1) is a special case of specializes to (3.1). On the other hand, by assumption, (3.2) has a solution, say $x$. Therefore, there exist $v_{1} \in \mathcal{G}_{1}, \ldots, v_{K_{2}} \in \mathcal{G}_{K_{2}}$ such that

$$
\begin{cases}z \in A x+\sum_{k=1}^{K_{2}} L_{k}^{*} v_{k}+\sum_{k=K_{2}+1}^{K} L_{k}^{*}\left(\left(B_{k} \square S_{k}\right)\left(L_{k} x-r_{k}\right)\right)+C x \\ \left(\forall k \in\left\{1, \ldots, K_{2}\right\}\right) \quad v_{k} \in\left(B_{k} \square S_{k}\right)\left(L_{k} x-r_{k}\right) .\end{cases}
$$

Therefore, in view of (1.9), there exist $y_{1} \in \mathcal{G}_{1}, \ldots, y_{K_{2}} \in \mathcal{G}_{K_{2}}$ such that

$$
\begin{cases}z \in A x+\sum_{k=1}^{K_{2}} L_{k}^{*} v_{k}+\sum_{k=K_{2}+1}^{K} L_{k}^{*}\left(\left(B_{k} \square S_{k}\right)\left(L_{k} x-r_{k}\right)\right)+C x \\ \left(\forall k \in\left\{1, \ldots, K_{2}\right\}\right) \quad y_{k} \in S_{k}^{-1} v_{k} \quad \text { and } \quad L_{k} x-y_{k}-r_{k} \in B_{k}^{-1} v_{k},\end{cases}
$$

which implies that

$$
\left\{\begin{array}{l}
z \in A x+\sum_{k=1}^{K_{2}} L_{k}^{*} v_{k}+\sum_{k=K_{2}+1}^{K} L_{k}^{*}\left(\left(B_{k} \square S_{k}\right)\left(L_{k} x-r_{k}\right)\right)+C x \\
\left(\forall k \in\left\{1, \ldots, K_{2}\right\}\right) \quad v_{k} \in S_{k} y_{k} \quad \text { and } \quad v_{k} \in B_{k}\left(L_{k} x-y_{k}-r_{k}\right),
\end{array}\right.
$$

and therefore that

$$
\left\{\begin{array}{l}
z \in A x+\sum_{k=1}^{K_{2}} L_{k}^{*}\left(B_{k}\left(L_{k} x-y_{k}-r_{k}\right)\right)+\sum_{k=K_{2}+1}^{K} L_{k}^{*}\left(\left(B_{k} \square S_{k}\right)\left(L_{k} x-r_{k}\right)\right)+C x \\
\left(\forall k \in\left\{1, \ldots, K_{2}\right\}\right) \quad 0 \in S_{k} y_{k}-B_{k}\left(L_{k} x-y_{k}-r_{k}\right) .
\end{array}\right.
$$

This shows that (3.5) possesses a solution. Next, upon defining

$$
(\forall n \in \mathbb{N}) \quad x_{1, n}=x_{n} \quad \text { and } \quad\left(\forall k \in\left\{1, \ldots, K_{2}\right\}\right) \quad \begin{cases}x_{k+1, n}=y_{k, n} ; \\ a_{1, k+1, n}=0, & \text { if } 1 \leqslant k \leqslant K_{1} ; \\ b_{1, k+1, n}=0, & \text { if } K_{1}+1 \leqslant k \leqslant K_{2} \\ c_{1, k+1, n}=0, & \text { if } 1 \leqslant k \leqslant K_{1},\end{cases}
$$

we see that (2.4) specializes to (3.4). Hence, in view of (3.6)-(3.7) and Theorem 2.4)(iii)(a) (iii)(d), there exist a solution $\left(\bar{x}, \overline{y_{1}}, \ldots, \overline{y_{K_{2}}}\right)$ to (3.5) and a solution $\left(\overline{v_{1}}, \ldots, \overline{v_{K}}\right)$ to (3.3) such that

$$
x_{n} \rightarrow \bar{x} \text { and }(\forall k \in\{1, \ldots, K\}) \quad v_{k, n} \rightarrow \overline{v_{k}}
$$


with

$$
\begin{array}{r}
z-\sum_{k=1}^{K} L_{k}^{*} \overline{v_{k}} \in A \bar{x}+C \bar{x}, \quad\left(\forall k \in\left\{1, \ldots, K_{2}\right\}\right) \quad\left\{\begin{array}{l}
L_{k} \bar{x}-\overline{y_{k}}-r_{k} \in B_{k}^{-1} \overline{v_{k}} \\
\overline{v_{k}} \in S_{k} \overline{y_{k}}
\end{array}\right. \\
\text { and }\left(\forall k \in\left\{K_{2}+1, \ldots, K\right\}\right) \quad L_{k} \bar{x}-r_{k} \in B_{k}^{-1} \overline{v_{k}}+S_{k}^{-1} \overline{v_{k}} .
\end{array}
$$

Since the strong convergence claims (ii) (iv) are immediate consequences of Theorem 2.4)(iii)(e)(iii)(f), it remains to show that $\bar{x}$ solves (3.2). We derive from (3.15) that, for every $k \in\left\{1, \ldots, K_{2}\right\}$, $L_{k} \bar{x}-\overline{y_{k}}-r_{k} \in B_{k}^{-1} \overline{v_{k}}$ and $\overline{y_{k}} \in S_{k}^{-1} \overline{v_{k}}$, and, for every $k \in\left\{K_{2}+1, \ldots, K\right\}, L_{k} \bar{x}-r_{k} \in B_{k}^{-1} \overline{v_{k}}+S_{k}^{-1} \overline{v_{k}}$. Altogether,

$$
(\forall k \in\{1, \ldots, K\}) \quad L_{k} \bar{x}-r_{k} \in\left(B_{k}^{-1}+S_{k}^{-1}\right) \overline{v_{k}}
$$

and, therefore,

$$
\sum_{k=1}^{K} L_{k}^{*} \overline{v_{k}} \in \sum_{k=1}^{K} L_{k}^{*}\left(\left(B_{k}^{-1}+S_{k}^{-1}\right)^{-1}\left(L_{k} \bar{x}-r_{k}\right)\right)=\sum_{k=1}^{K} L_{k}^{*}\left(\left(B_{k} \square S_{k}\right)\left(L_{k} \bar{x}-r_{k}\right)\right) .
$$

Thus, since (3.15) also asserts that $z-\sum_{k=1}^{K} L_{k}^{*} \overline{v_{k}} \in A \bar{x}+C \bar{x}$, we conclude that $\bar{x}$ solves (3.2).

Remark 3.3 Problem 3.1 encompasses more general scenarios than that of [19], which corresponds to the case when $K_{1}=K_{2}=0$, i.e., when all the operators $\left(D_{k}^{-1}\right)_{1 \leqslant k \leqslant K}$ are restricted to be Lipschitzian. This extension has been made possible by reformulating the original primal problem (3.2), which involves only one variable, as the extended primal problem (3.5), in which we added $K_{2}$ auxiliary variables. We also note that Algorithm (3.4) uses all the single-valued operators present in Problem 3.1, including $\left(S_{k}\right)_{K_{1}+1 \leqslant k \leqslant K_{2}}$ and $\left(S_{k}^{-1}\right)_{K_{2}+1 \leqslant k \leqslant K}$, through explicit steps.

\section{Relaxation of inconsistent common zero problems}

A common problem in nonlinear analysis is to find a common zero of maximally monotone operators $A$ and $\left(B_{k}\right)_{1 \leqslant k \leqslant K}$ acting on a real Hilbert space $\mathcal{H}[16,22,32]$, i.e.,

$$
\text { find } \bar{x} \in \mathcal{H} \text { such that } 0 \in A \bar{x} \cap \bigcap_{k=1}^{K} B_{k} \bar{x} \text {. }
$$

In many situations, this problem may be inconsistent (see [18] and the references therein) and it must be approximated. We study the following relaxation of (4.1), together with its dual problem.

Problem 4.1 Let $\mathcal{H}$ be a real Hilbert space, let $K$ be a strictly positive integer, let $A: \mathcal{H} \rightarrow 2^{\mathcal{H}}$ be maximally monotone, and, for every $k \in\{1, \ldots, K\}$, let $S_{k}: \mathcal{H} \rightarrow 2^{\mathcal{H}}$ be a maximally monotone operator such that $S_{k}^{-1}$ is at most single-valued and strictly monotone, with $S_{k}^{-1} 0=\{0\}$. It is assumed that the inclusion

$$
\text { find } \bar{x} \in \mathcal{H} \text { such that } 0 \in A \bar{x}+\sum_{k=1}^{K}\left(B_{k} \square S_{k}\right) \bar{x}
$$


possesses at least one solution. Solve (4.2) together with the dual problem

find $\overline{u_{1}} \in \mathcal{H}, \ldots, \overline{u_{K}} \in \mathcal{H}$ such that

$$
(\forall k \in\{1, \ldots, K\}) \quad 0 \in-A^{-1}\left(-\sum_{l=1}^{K} \overline{u_{l}}\right)+B_{k}^{-1} \overline{u_{k}}+S_{k}^{-1} \overline{u_{k}} .
$$

First, we justify the fact that (4.2) is indeed a relaxation of (4.1).

Proposition 4.2 In the setting of Problem 4.1 set $Z=(\operatorname{zer} A) \cap \bigcap_{k=1}^{K}$ zer $B_{k}$ and suppose that $Z \neq \varnothing$. Then the set of solutions to the primal problem (4.2) is $Z$.

Proof. It is clear that every point in $Z$ solves (4.2). Conversely, let $x$ be a solution to (4.2) and let $z \in Z$. We first note that the operators $\left(B_{k} \square S_{k}\right)_{1 \leqslant k \leqslant K}$ are at most single-valued. Indeed, let $k \in$ $\{1, \ldots, K\}$ and let $(y, p)$ and $(y, q)$ be in $\operatorname{gra}\left(B_{k} \square S_{k}\right)$. Then we must show that $p=q$. We have $p=$ $\left(B_{k} \square S_{k}\right) y \Leftrightarrow y \in B_{k}^{-1} p+S_{k}^{-1} p \Leftrightarrow y-S_{k}^{-1} p \in B_{k}^{-1} p$. Likewise, $y-S_{k}^{-1} q \in B_{k}^{-1} q$ and, by monotonicity of $B_{k},-\left\langle p-q \mid S_{k}^{-1} p-S_{k}^{-1} q\right\rangle=\left\langle p-q \mid\left(y-S_{k}^{-1} p\right)-\left(y-S_{k}^{-1} q\right)\right\rangle \geqslant 0$. Consequently, by strict monotonicity of $S_{k}^{-1},\left\langle p-q \mid S_{k}^{-1} p-S_{k}^{-1} q\right\rangle=0$ and $p=q$. Hence, since $x$ solves (4.2), there exists $\left(p_{k}\right)_{0 \leqslant k \leqslant K} \in \mathcal{H}^{K+1}$ such that

$$
\sum_{k=0}^{K} p_{k}=0, \quad p_{0} \in A x, \quad \text { and } \quad(\forall k \in\{1, \ldots, K\}) \quad p_{k}=\left(B_{k} \square S_{k}\right) x .
$$

Therefore, we have

$$
p_{0} \in A x, 0 \in A z, \quad \text { and } \quad(\forall k \in\{1, \ldots, K\}) \quad p_{k} \in B_{k}\left(x-S_{k}^{-1} p_{k}\right) \quad \text { and } \quad 0 \in B_{k} z,
$$

and, by monotonicity of the operators $A$ and $\left(B_{k}\right)_{1 \leqslant k \leqslant K}$,

$$
\left\langle x-z \mid p_{0}\right\rangle \geqslant 0 \quad \text { and } \quad(\forall k \in\{1, \ldots, K\}) \quad\left\langle x-S_{k}^{-1} p_{k}-z \mid p_{k}\right\rangle \geqslant 0 .
$$

Hence, since $\sum_{k=0}^{K} p_{k}=0$, it follows from the monotonicity of the operators $\left(S_{k}^{-1}\right)_{1 \leqslant k \leqslant K}$ that

$$
\begin{aligned}
0 & \geqslant-\sum_{k=1}^{K}\left\langle p_{k}-0 \mid S_{k}^{-1} p_{k}-S_{k}^{-1} 0\right\rangle \\
& =\sum_{k=0}^{K}\left\langle x-z \mid p_{k}\right\rangle-\sum_{k=1}^{K}\left\langle S_{k}^{-1} p_{k} \mid p_{k}\right\rangle \\
& =\left\langle x-z \mid p_{0}\right\rangle+\sum_{k=1}^{K}\left\langle x-S_{k}^{-1} p_{k}-z \mid p_{k}\right\rangle \\
& \geqslant 0 .
\end{aligned}
$$

Thus, $\sum_{k=1}^{K}\left\langle p_{k}-0 \mid S_{k}^{-1} p_{k}-S_{k}^{-1} 0\right\rangle=0$ and, therefore,

$$
(\forall k \in\{1, \ldots, K\}) \quad\left\langle p_{k}-0 \mid S_{k}^{-1} p_{k}-S_{k}^{-1} 0\right\rangle=0 .
$$

The strict monotonicity of the operators $\left(S_{k}^{-1}\right)_{1 \leqslant k \leqslant K}$ implies that for every $k \in\{1, \ldots, K\} p_{k}=0$, i.e., $x \in B_{k}^{-1} p_{k}+S_{k}^{-1} p_{k}=B_{k}^{-1} 0+S_{k}^{-1} 0=B_{k}^{-1} 0$. In turn, $p_{0}=-\sum_{k=1}^{K} p_{k}=0$, i.e., $x \in A^{-1} 0$. Altogether, $x \in Z$. $\square$ 
Remark 4.3 Suppose that in Problem 4.1 we set, for every $k \in\{1, \ldots, K\}, S_{k}=\gamma_{k}^{-1}$ Id where $\gamma_{k} \in$ ]0, $+\infty$ [, i.e., $B_{k} \square S_{k}={ }^{\gamma_{k}} B_{k}$ is the Yosida approximation of $B_{k}$ of index $\gamma_{k}$ [8, Proposition 23.6(ii)]. Then (4.2) reduces to the setting investigated in [17, Section 6.3], namely

$$
\text { find } \bar{x} \in \mathcal{H} \text { such that } 0 \in A \bar{x}+\sum_{k=1}^{K} \gamma_{k} B_{k} \bar{x},
$$

which itself covers the frameworks of [10, 18, 35, 37] and the references therein. In this case, Proposition 4.2 specializes to [17, Proposition 6.10]. Now let us further specialize to the case when $\mathcal{H}=\mathbb{R}^{N}, A=0$, and

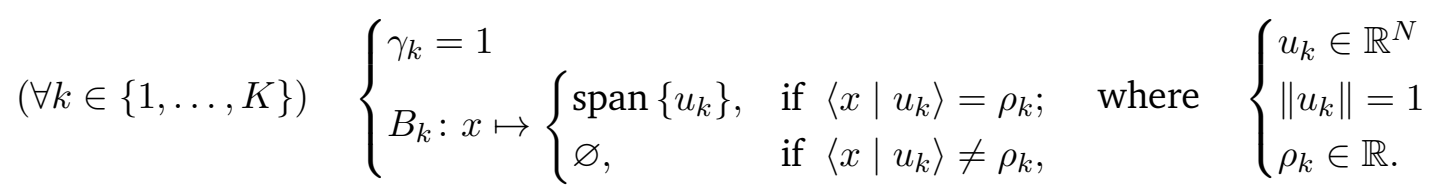

Then (4.1) amounts to solving the system of linear equalities

$$
\text { find } \bar{x} \in \mathbb{R}^{N} \text { such that }(\forall k \in\{1, \ldots, K\}) \quad\left\langle\bar{x} \mid u_{k}\right\rangle=\rho_{k},
$$

whereas (4.2) amounts to solving the least-squares problem

$$
\underset{x \in \mathbb{R}^{N}}{\operatorname{minimize}} \sum_{k=1}^{m}\left|\left\langle x \mid u_{k}\right\rangle-\rho_{k}\right|^{2}
$$

The idea of relaxing (4.11) to (4.12) is due to Legendre [31] and Gauss [26].

To solve Problem 4.1, we use Proposition 3.2 to derive the following algorithm.

Proposition 4.4 Consider the setting of Problem 4.1 Let $\left(b_{1,1, n}\right)_{n \in \mathbb{N}}$ and, for every $k \in\{1, \ldots, K\}$, $\left(b_{1, k+1, n}\right)_{n \in \mathbb{N}}$ and $\left(b_{2, k, n}\right)_{n \in \mathbb{N}}$ be absolutely summable sequences in $\mathcal{H}$. Let $x_{0} \in \mathcal{H},\left(y_{k, 0}\right)_{1 \leqslant k \leqslant K} \in \mathcal{H}^{K}$, $\left(v_{k, 0}\right)_{1 \leqslant k \leqslant K} \in \mathcal{H}^{K}$, and $\left.\varepsilon \in\right] 0,1 /(\sqrt{K+1}+1)\left[\right.$, let $\left(\gamma_{n}\right)_{n \in \mathbb{N}}$ be a sequence in $[\varepsilon,(1-\varepsilon) / \sqrt{K+1}]$, and set

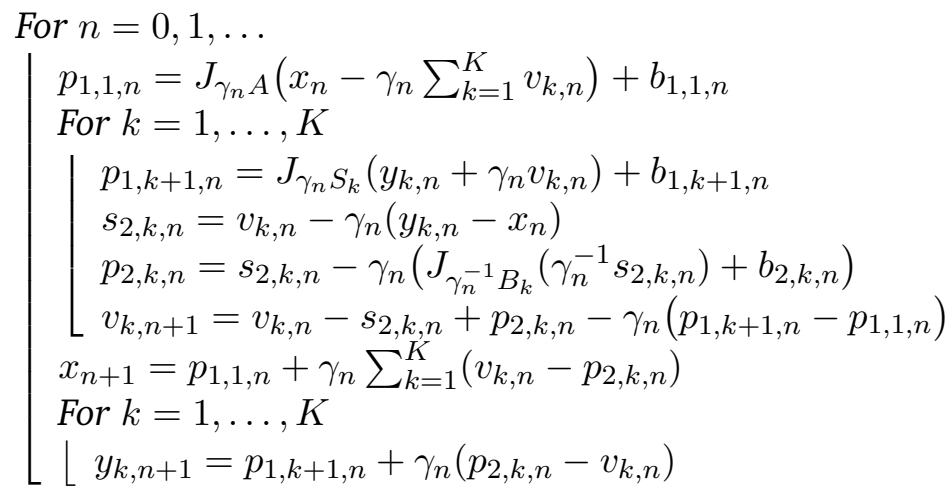

Then the following hold for some solution $\bar{x}$ to (4.2) and some solution $\left(\overline{v_{1}}, \ldots, \overline{v_{K}}\right)$ to (4.3).

(i) $x_{n} \rightarrow \bar{x}$ and $(\forall k \in\{1, \ldots, K\}) v_{k, n} \rightarrow \overline{v_{k}}$.

(ii) Suppose that $A$ is uniformly monotone at $\bar{x}$. Then $x_{n} \rightarrow \bar{x}$. 
(iii) Suppose that, for some $l \in\{1, \ldots, K\}, B_{l}$ is couniformly monotone at $\bar{v}_{l}$. Then $v_{l, n} \rightarrow \bar{v}_{l}$.

Proof. Problem 4.1 is a special case of Problem 3.1 with $K_{1}=K_{2}=K, z=0, C=0, \mu=0$, $\beta=\sqrt{K+1}$, and $(\forall k \in\{1, \ldots, K\}) \mathcal{G}_{k}=\mathcal{H}, L_{k}=$ Id, and $r_{k}=0$. In this context, (3.4) can be reduced to (4.13), and the claims therefore follow from Proposition 3.2. $\mathrm{C}$

Remark 4.5 For brevity, we have presented an algorithm for solving Problem 4.1 in its general form. However, if some of the operators $\left(S_{k}\right)_{1 \leqslant k \leqslant K}$ or their inverses are Lipschitzian, we can apply Proposition 3.2 with $K_{1} \neq K$ and/or $K_{2} \neq K$ to obtain a more efficient algorithm in which each Lipschitzian operator is used through an explicit step, rather than through its resolvent.

\section{Multivariate structured convex minimization problems}

We derive from Theorem 2.4 a primal-dual minimization algorithm for multivariate convex minimization problems involving infimal convolutions and composite functions.

Problem 5.1 Let $m$ and $K$ be strictly positive integers, let $\left(\mathcal{H}_{i}\right)_{1 \leqslant i \leqslant m}$ and $\left(\mathcal{G}_{k}\right)_{1 \leqslant k \leqslant K}$ be real Hilbert spaces, let $\left(\mu_{i}\right)_{1 \leqslant i \leqslant m} \in\left[0,+\infty\left[^{m}\right.\right.$, and let $\left.\left(\nu_{k}\right)_{1 \leqslant i \leqslant K} \in\right] 0,+\infty\left[^{K}\right.$. For every $i \in\{1, \ldots, m\}$ and $k \in\{1, \ldots, K\}$, let $h_{i}: \mathcal{H}_{i} \rightarrow \mathbb{R}$ be convex and differentiable and such that $\nabla h_{i}$ is $\mu_{i^{-}}$ Lipschitzian, let $f_{i} \in \Gamma_{0}\left(\mathcal{H}_{i}\right)$, let $g_{k} \in \Gamma_{0}\left(\mathcal{G}_{k}\right)$, let $\ell_{k} \in \Gamma_{0}\left(\mathcal{G}_{k}\right)$ be $1 / \nu_{k}$-strongly convex, let $z_{i} \in \mathcal{H}_{i}$, let $r_{k} \in \mathcal{G}_{k}$, and let $L_{k i} \in \mathcal{B}\left(\mathcal{H}_{i}, \mathcal{G}_{k}\right)$. Set $\beta=\max \left\{\max _{1 \leqslant i \leqslant m} \mu_{i}, \max _{1 \leqslant k \leqslant K} \nu_{k}\right\}+\sqrt{\lambda}>0$, where $\lambda \in\left[\sup _{\sum_{i=1}^{m}\left\|x_{i}\right\|^{2} \leqslant 1} \sum_{k=1}^{K}\left\|\sum_{i=1}^{m} L_{k i} x_{i}\right\|^{2},+\infty[\right.$, and assume that

$$
(\forall i \in\{1, \ldots, m\}) \quad z_{i} \in \operatorname{ran}\left(\partial f_{i}+\sum_{k=1}^{K} L_{k i}^{*} \circ\left(\partial g_{k} \square \partial \ell_{k}\right) \circ\left(\sum_{j=1}^{m} L_{k j} \cdot-r_{k}\right)+\nabla h_{i}\right) .
$$

Solve the primal problem

$$
\underset{x_{1} \in \mathcal{H}_{1}, \ldots, x_{m} \in \mathcal{H}_{m}}{\operatorname{minimize}} \sum_{i=1}^{m} f_{i}\left(x_{i}\right)+\sum_{k=1}^{K}\left(g_{k} \square \ell_{k}\right)\left(\sum_{i=1}^{m} L_{k i} x_{i}-r_{k}\right)+\sum_{i=1}^{m}\left(h_{i}\left(x_{i}\right)-\left\langle x_{i} \mid z_{i}\right\rangle\right),
$$

together with the dual problem

$$
\underset{v_{1} \in \mathcal{G}_{1}, \ldots, v_{K} \in \mathcal{G}_{K}}{\operatorname{minimize}} \sum_{i=1}^{m}\left(f_{i}^{*} \square h_{i}^{*}\right)\left(z_{i}-\sum_{k=1}^{K} L_{k i}^{*} v_{k}\right)+\sum_{k=1}^{K}\left(g_{k}^{*}\left(v_{k}\right)+\ell_{k}^{*}\left(v_{k}\right)+\left\langle v_{k} \mid r_{k}\right\rangle\right) .
$$

Remark 5.2 Problem 5.1 extends significantly the multivariate minimization framework of [3, 12]. There, $\left(h_{i}\right)_{1 \leqslant i \leqslant m}$ were the zero function, $\left(\ell_{k}\right)_{1 \leqslant k \leqslant K}$ were the function $\iota_{\{0\}}$, and $\left(g_{k}\right)_{1 \leqslant k \leqslant K}$ were differentiable everywhere with a Lipschitzian gradient. Finally, no dual problem was considered.

Proposition 5.3 Consider the setting of Problem 5.1. Suppose that (5.2) has a solution, and set

$$
E=\left\{\left(\sum_{i=1}^{m} L_{k i} x_{i}-y_{k}\right)_{1 \leqslant k \leqslant K} \mid\left\{\begin{array}{l}
(\forall i \in\{1, \ldots, m\}) x_{i} \in \operatorname{dom} f_{i} \\
(\forall k \in\{1, \ldots, K\}) y_{k} \in \operatorname{dom} g_{k}+\operatorname{dom} \ell_{k}
\end{array}\right\} .\right.
$$

Then (5.1) is satisfied in each of the following cases. 
(i) $\left(r_{k}\right)_{1 \leqslant k \leqslant K} \in \operatorname{sri} E$.

(ii) $E-\left(r_{k}\right)_{1 \leqslant k \leqslant K}$ is a closed vector subspace.

(iii) For every $i \in\{1, \ldots, m\}$, $f_{i}$ is real-valued and, for every $k \in\{1, \ldots, K\}$, the operator $\bigoplus_{j=1}^{m} \mathcal{H}_{j} \rightarrow$ $\mathcal{G}_{k}:\left(x_{j}\right)_{1 \leqslant j \leqslant m} \mapsto \sum_{j=1}^{m} L_{k j} x_{j}$ is surjective.

(iv) For every $k \in\{1, \ldots, K\}, g_{k}$ or $\ell_{k}$ is real-valued.

(v) $\left(\mathcal{H}_{i}\right)_{1 \leqslant i \leqslant m}$ and $\left(\mathcal{G}_{k}\right)_{1 \leqslant k \leqslant K}$ are finite-dimensional, and $(\forall i \in\{1, \ldots, m\})\left(\exists x_{i} \in \operatorname{ridom} f_{i}\right)(\forall k \in$ $\{1, \ldots, K\}) \sum_{i=1}^{m} L_{k i} x_{i}-r_{k} \in \operatorname{ridom} g_{k}+$ ridom $\ell_{k}$.

Proof. Define $\mathcal{H}$ and $\mathcal{G}$ as in (2.5), and $\boldsymbol{L}, \boldsymbol{z}$, and $\boldsymbol{r}$ as in (2.6). Set

$$
\left\{\begin{array}{l}
\boldsymbol{f}: \mathcal{H} \rightarrow]-\infty,+\infty]: \boldsymbol{x} \mapsto \sum_{i=1}^{m} f_{i}\left(x_{i}\right) \quad \text { and } \quad \boldsymbol{h}: \mathcal{H} \rightarrow \mathbb{R}: \boldsymbol{x} \mapsto \sum_{i=1}^{m} h_{i}\left(x_{i}\right), \\
\left.\left.\boldsymbol{g}: \mathcal{G} \rightarrow]-\infty,+\infty]: \boldsymbol{y} \mapsto \sum_{k=1}^{K} g_{k}\left(y_{k}\right) \quad \text { and } \quad \boldsymbol{\ell}: \mathcal{G} \rightarrow\right]-\infty,+\infty\right]: \boldsymbol{y} \mapsto \sum_{k=1}^{K} \ell_{k}\left(y_{k}\right) .
\end{array}\right.
$$

Then (5.4) and [8, Proposition 12.6(ii)] yield

$$
\begin{aligned}
E & =\{\boldsymbol{L} \boldsymbol{x}-\boldsymbol{y} \mid \boldsymbol{x} \in \operatorname{dom} \boldsymbol{f} \text { and } \boldsymbol{y} \in \operatorname{dom} \boldsymbol{g}+\operatorname{dom} \boldsymbol{\ell}\} \\
& =\boldsymbol{L}(\operatorname{dom} \boldsymbol{f})-(\operatorname{dom} \boldsymbol{g}+\operatorname{dom} \boldsymbol{\ell}) \\
& =\boldsymbol{L}(\operatorname{dom}(\boldsymbol{f}+\boldsymbol{h}-\langle\cdot \mid \boldsymbol{z}\rangle))-\operatorname{dom}(\boldsymbol{g} \square \boldsymbol{\ell}) .
\end{aligned}
$$

(i): Since the functions $\left(\ell_{k}\right)_{1 \leqslant k \leqslant K}$ are strongly convex, so is $\ell$. Hence, dom $\ell^{*}=\mathcal{G}$ [8, Propositions 11.16 and 14.15] and therefore [8, Propositions 15.7(iv) and 24.27] imply that $\partial \boldsymbol{g} \square \partial \boldsymbol{\ell}=\partial(\boldsymbol{g} \square \boldsymbol{\ell})$ and $\boldsymbol{g} \square \boldsymbol{\ell} \in \Gamma_{0}(\mathcal{G})$. On the other hand, (5.7) yields $\mathbf{0} \in \operatorname{sri}(\boldsymbol{L}(\operatorname{dom}(\boldsymbol{f}+\boldsymbol{h}-$ $\langle\cdot \mid \boldsymbol{z}\rangle))-\operatorname{dom}(\boldsymbol{g} \square \boldsymbol{\ell})(\cdot-\boldsymbol{r}))$. Thus, we derive from [8, Theorem 16.37(i)] that

$$
\begin{aligned}
\partial \boldsymbol{f}+\boldsymbol{L}^{*} \circ\left(\partial \boldsymbol{g} \square \partial \boldsymbol{\ell}_{k}\right) \circ(\boldsymbol{L} \cdot-\boldsymbol{r})+\nabla \boldsymbol{h}-\boldsymbol{z} & =\partial(\boldsymbol{f}+\boldsymbol{h}-\langle\cdot \mid \boldsymbol{z}\rangle)+\boldsymbol{L}^{*} \circ \partial(\boldsymbol{g} \square \boldsymbol{\ell}) \circ(\boldsymbol{L} \cdot-\boldsymbol{r}) \\
& =\partial(\boldsymbol{f}+\boldsymbol{h}-\langle\cdot \mid \boldsymbol{z}\rangle+(\boldsymbol{g} \square \boldsymbol{\ell}) \circ(\boldsymbol{L} \cdot-\boldsymbol{r})) .
\end{aligned}
$$

Since (5.2) has a solution and is equivalent to minimizing $\boldsymbol{f}+\boldsymbol{h}-\langle\cdot \mid \boldsymbol{z}\rangle+(\boldsymbol{g} \square \boldsymbol{\ell}) \circ(\boldsymbol{L} \cdot-r)$ over $\boldsymbol{H}$, Fermat's rule [8, Theorem 16.2] implies that $\mathbf{0} \in \operatorname{ran} \partial(\boldsymbol{f}+\boldsymbol{h}-\langle\cdot \mid \boldsymbol{z}\rangle+(\boldsymbol{g} \square \boldsymbol{\ell}) \circ(\boldsymbol{L} \cdot-\boldsymbol{r}))$. Hence (5.8) yields $\boldsymbol{z} \in \operatorname{ran}\left(\partial \boldsymbol{f}+\boldsymbol{L}^{*} \circ\left(\partial \boldsymbol{g} \square \partial \boldsymbol{\ell}_{k}\right) \circ(\boldsymbol{L} \cdot-\boldsymbol{r})+\nabla \boldsymbol{h}\right)$ and we conclude that (5.1) is satisfied.

(ii) $\Rightarrow$ (i): [8, Proposition 6.19(i)].

(iii) $\Rightarrow$ (i); We have $\boldsymbol{L}(\operatorname{dom} \boldsymbol{f})=\boldsymbol{L}(\mathcal{H})=\mathcal{G}$. Hence, (5.6) yields $E=\mathcal{G}$.

(iv) $\Rightarrow$ (i); We have $\operatorname{dom} \boldsymbol{g}+\operatorname{dom} \boldsymbol{\ell}=\mathcal{G}$. Hence, (5.6) yields $E=\mathcal{G}$.

(v) $\Rightarrow$ (i); Since $\operatorname{dim} \mathcal{G}<+\infty$, sri $E=\operatorname{ri} E$. On the other hand, by (5.6) and [8, Corollary 6.15],

$$
\text { ri } E=\operatorname{ri}(\boldsymbol{L}(\operatorname{dom} \boldsymbol{f})-\operatorname{dom} \boldsymbol{g}-\operatorname{dom} \boldsymbol{\ell})=\boldsymbol{L}(\operatorname{ridom} \boldsymbol{f})-\operatorname{ridom} \boldsymbol{g}-\operatorname{ridom} \boldsymbol{\ell} .
$$

Thus, $\boldsymbol{r} \in \operatorname{sri} E \Leftrightarrow\left(\exists \boldsymbol{x} \in \operatorname{ridom} \boldsymbol{f}=\times_{i=1}^{m}\right.$ ri dom $\left.f_{i}\right) \boldsymbol{L} \boldsymbol{x}-\boldsymbol{r} \in \operatorname{ridom} \boldsymbol{g}+$ ridom $\boldsymbol{\ell}=\times_{k=1}^{K}\left(\right.$ ridom $g_{k}+$ ri dom $\left.\ell_{k}\right)$.

Proposition 5.4 Consider the setting of Problem [5.1 For every $i \in\{1, \ldots, m\}$, let $\left(a_{1, i, n}\right)_{n \in \mathbb{N}}$, $\left(b_{1, i, n}\right)_{n \in \mathbb{N}}$, and $\left(c_{1, i, n}\right)_{n \in \mathbb{N}}$ be absolutely summable sequences in $\mathcal{H}_{i}$ and, for every $k \in\{1, \ldots, K\}$, let $\left(a_{2, k, n}\right)_{n \in \mathbb{N}},\left(b_{2, k, n}\right)_{n \in \mathbb{N}}$, and $\left(c_{2, k, n}\right)_{n \in \mathbb{N}}$ be absolutely summable sequences in $\mathcal{G}_{k}$. Furthermore, let 
$x_{1,0} \in \mathcal{H}_{1}, \ldots, x_{m, 0} \in \mathcal{H}_{m}, v_{1,0} \in \mathcal{G}_{1}, \ldots, v_{K, 0} \in \mathcal{G}_{K}$, let $\left.\varepsilon \in\right] 0,1 /(\beta+1)\left[\right.$, let $\left(\gamma_{n}\right)_{n \in \mathbb{N}}$ be a sequence in $[\varepsilon,(1-\varepsilon) / \beta]$, and set

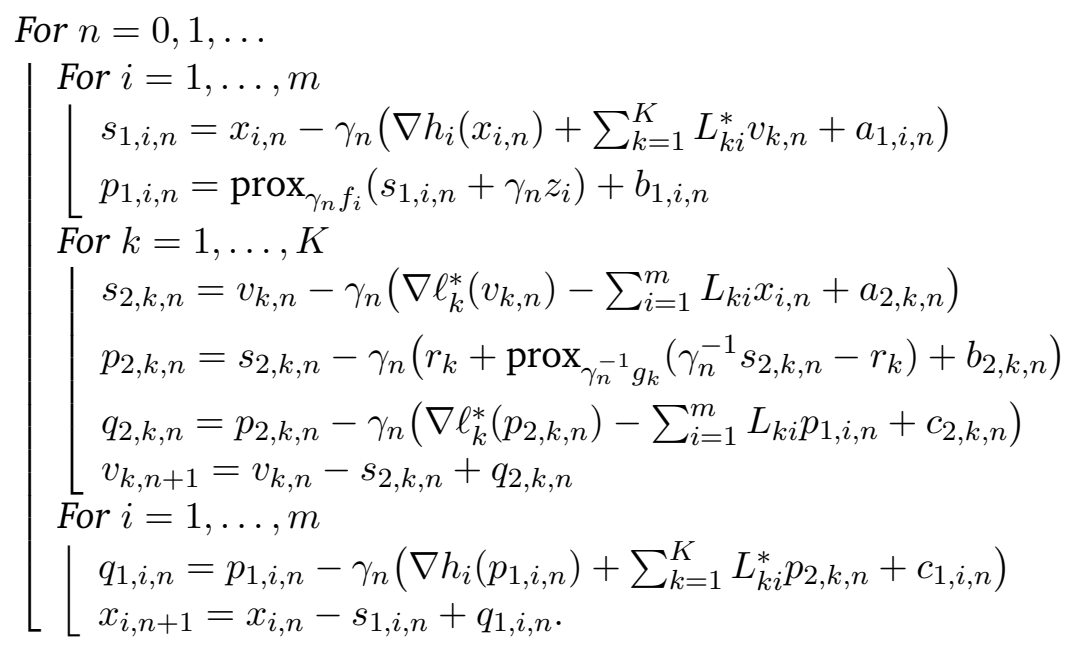

Then the following hold.

(i) $(\forall i \in\{1, \ldots, m\}) \sum_{n \in \mathbb{N}}\left\|x_{i, n}-p_{1, i, n}\right\|^{2}<+\infty$, and $(\forall k \in\{1, \ldots, K\}) \sum_{n \in \mathbb{N}}\left\|v_{k, n}-p_{2, k, n}\right\|^{2}<+\infty$.

(ii) There exist a solution $\left(\overline{x_{1}}, \ldots, \overline{x_{m}}\right)$ to (5.2) and a solution $\left(\overline{v_{1}}, \ldots, \overline{v_{K}}\right)$ to (5.3) such that the following hold.

(a) $(\forall i \in\{1, \ldots, m\}) x_{i, n} \rightarrow \overline{x_{i}}$ and $z_{i}-\sum_{k=1}^{K} L_{k i}^{*} \overline{v_{k}} \in \partial f_{i}\left(\overline{x_{i}}\right)+\nabla h_{i}\left(\overline{x_{i}}\right)$.

(b) $(\forall k \in\{1, \ldots, K\}) v_{k, n} \rightarrow \overline{v_{k}}$ and $\sum_{i=1}^{m} L_{k i} \overline{x_{i}}-r_{k} \in \partial g_{k}^{*}\left(\overline{v_{k}}\right)+\nabla \ell_{k}^{*}\left(\overline{v_{k}}\right)$.

(c) Suppose that, for some $j \in\{1, \ldots, m\}$, $f_{j}$ or $h_{j}$ is uniformly convex at $\overline{x_{j}}$. Then $x_{j, n} \rightarrow \overline{x_{j}}$.

(d) Suppose that, for some $l \in\{1, \ldots, K\}$, $g_{l}^{*}$ or $\ell_{l}^{*}$ is uniformly convex at $\overline{v_{l}}$. Then $v_{l, n} \rightarrow \overline{v_{l}}$.

Proof. Set

$$
\begin{cases}(\forall i \in\{1, \ldots, m\}) & A_{i}=\partial f_{i} \quad \text { and } \quad C_{i}=\nabla h_{i} \\ (\forall k \in\{1, \ldots, K\}) & B_{k}=\partial g_{k} \quad \text { and } \quad D_{k}=\partial \ell_{k} .\end{cases}
$$

It follows from [8, Proposition 17.10] that the operators $\left(C_{i}\right)_{1 \leqslant i \leqslant m}$ are monotone, and from [8, Theorem 20.40] that the operators $\left(A_{i}\right)_{1 \leqslant i \leqslant m},\left(B_{k}\right)_{1 \leqslant k \leqslant m}$, and $\left(D_{k}\right)_{1 \leqslant k \leqslant K}$ are maximally monotone. Moreover, for every $k \in\{1, \ldots, K\}$, we derive from [8, Corollary 13.33 and Theorem 18.15] that $\ell_{k}^{*}$ is Fréchet differentiable on $\mathcal{G}_{k}$ and $\nabla \ell_{k}^{*}$ is $\nu_{k}$-Lipschitzian, and from [8, Corollary 16.24 and Proposition 17.26(i)] that $D_{k}^{-1}=\left(\partial \ell_{k}\right)^{-1}=\partial \ell_{k}^{*}=\left\{\nabla \ell_{k}^{*}\right\}$. On the other hand, (5.1) implies that (1.2) possesses a solution, and (1.12) implies that (5.10) is a special case of (2.4). We also recall that the uniform convexity of a function $\varphi \in \Gamma_{0}(\mathcal{H})$ at $x \in \operatorname{dom} \partial \varphi$ implies the uniform monotonicity of $\partial \varphi$ at $x$ [46, Section 3.4]. Altogether, the claims will follow at once from Theorem 2.4 provided we show that, in the setting of (5.1) and (5.11), (1.2) becomes (5.2) and (1.3) becomes (5.3). To this end, let us first observe that since, for every $k \in\{1, \ldots, K\}$, dom $\ell_{k}^{*}=\mathcal{G}_{k}$, [8, Proposition 24.27] yields

$$
(\forall k \in\{1, \ldots, K\}) \quad B_{k} \square D_{k}=\partial g_{k} \square \partial \ell_{k}=\partial\left(g_{k} \square \ell_{k}\right),
$$


while [8, Corollaries 16.24 and 16.38(iii)] yield

$$
(\forall k \in\{1, \ldots, K\}) \quad B_{k}^{-1}+D_{k}^{-1}=\partial g_{k}^{*}+\left\{\nabla \ell_{k}^{*}\right\}=\partial\left(g_{k}^{*}+\ell_{k}^{*}\right) .
$$

Likewise, using [8, Theorem 15.3], we obtain

$$
(\forall i \in\{1, \ldots, m\}) \quad\left(A_{i}+C_{i}\right)^{-1}=\left(\partial f_{i}+\nabla h_{i}\right)^{-1}=\left(\partial\left(f_{i}+h_{i}\right)\right)^{-1}=\partial\left(f_{i}+h_{i}\right)^{*}=\partial\left(f_{i}^{*} \square h_{i}^{*}\right) .
$$

Now let us define $\mathcal{H}$ and $\mathcal{G}$ as in (2.5), $\boldsymbol{L}, \boldsymbol{z}$, and $\boldsymbol{r}$ as in (2.6), and $\boldsymbol{f}, \boldsymbol{h}, \boldsymbol{g}$, and $\boldsymbol{\ell}$ as in (5.5). We derive from (5.11), (5.12), [8, Corollary 16.38(iii), Propositions 16.5(ii), 16.8, and 17.26(i)], and Fermat's rule [8, Theorem 16.2] that, for every $\boldsymbol{x}=\left(x_{i}\right)_{1 \leqslant i \leqslant m} \in \mathcal{H}$,

$$
\begin{aligned}
\boldsymbol{x} \text { solves (1.2) } & \Leftrightarrow(\forall i \in\{1, \ldots, m\}) \quad 0 \in \partial f_{i}\left(x_{i}\right)+\sum_{k=1}^{K} L_{k i}^{*}\left(\partial\left(g_{k} \square \ell_{k}\right)\left(\sum_{j=1}^{m} L_{k j} x_{j}-r_{k}\right)\right) \\
& \quad+\nabla h_{i}\left(x_{i}\right)-z_{i} \\
\Leftrightarrow & \mathbf{0} \in \partial \boldsymbol{f}(\boldsymbol{x})+\boldsymbol{L}^{*}(\partial(\boldsymbol{g} \square \boldsymbol{\ell})(\boldsymbol{L} \boldsymbol{x}-\boldsymbol{r}))+\nabla(\boldsymbol{h}-\langle\cdot \mid \boldsymbol{z}\rangle)(\boldsymbol{x}) \\
\Rightarrow & \mathbf{0} \in \partial(\boldsymbol{f}+(\boldsymbol{g} \square \boldsymbol{\ell}) \circ(\boldsymbol{L} \cdot-\boldsymbol{r}))+\boldsymbol{h}-\langle\cdot \mid \boldsymbol{z}\rangle)(\boldsymbol{x}) \\
& \Leftrightarrow \boldsymbol{x} \text { solves (5.2). }
\end{aligned}
$$

Next, let $\boldsymbol{v}=\left(v_{k}\right)_{1 \leqslant k \leqslant K} \in \mathcal{G}$. Then we derive from (5.13), (5.14), and the same subdifferential calculus rules as above that

$$
\begin{aligned}
& \boldsymbol{v} \text { solves (1.3) } \Leftrightarrow(\forall k \in\{1, \ldots, K\}) \quad 0 \in-\sum_{i=1}^{m} L_{k i}\left(\partial\left(f_{i}^{*} \square h_{i}^{*}\right)\left(z_{i}-\sum_{l=1}^{K} L_{l i}^{*} v_{l}\right)\right) \\
& +\partial\left(g_{k}^{*}+\ell_{k}^{*}+\left\langle\cdot \mid r_{k}\right\rangle\right)\left(v_{k}\right) \\
& \Leftrightarrow \mathbf{0} \in-\boldsymbol{L}\left(\partial\left(\boldsymbol{f}^{*} \square \boldsymbol{h}^{*}\right)\left(\boldsymbol{z}-\boldsymbol{L}^{*} \boldsymbol{v}\right)\right)+\partial\left(\boldsymbol{g}^{*}+\boldsymbol{\ell}^{*}+\langle\cdot \mid \boldsymbol{r}\rangle\right)(\boldsymbol{v}) \\
& \Rightarrow \mathbf{0} \in \partial\left(\left(\boldsymbol{f}^{*} \square \boldsymbol{h}^{*}\right) \circ\left(\boldsymbol{z}-\boldsymbol{L}^{*} \cdot\right)+\boldsymbol{g}^{*}+\boldsymbol{\ell}^{*}+\langle\cdot \mid \boldsymbol{r}\rangle\right)(\boldsymbol{v}) \\
& \Leftrightarrow \boldsymbol{v} \text { solves (5.3), }
\end{aligned}
$$

which completes the proof. $\square$

Remark 5.5 Proposition 5.4 provides a framework that captures and suggests extensions of multivariate and/or infimal convolution variational formulations found in areas such as partial differential equations [4], machine learning [6], and image recovery [14, 15, 38].

\section{Univariate structured convex minimization problems}

Minimization problems involving a single primal variable can be obtained by setting $m=1$ in Problem 5.1. However, this approach imposes that infimal convolutions be performed exclusively with strongly convex functions. We use a different strategy relying on Proposition 3.2, which leads to a formulation allowing for infimal convolutions with general lower semicontinuous convex functions.

Problem 6.1 Let $\mathcal{H}$ be a real Hilbert space, let $K_{1}, K_{2}$, and $K$ be integers such that $0 \leqslant K_{1} \leqslant$ $K_{2} \leqslant K \geqslant 1$, let $z \in \mathcal{H}$, let $f \in \Gamma_{0}(\mathcal{H})$, and let $h: \mathcal{H} \rightarrow \mathbb{R}$ be convex and differentiable and such that $\nabla h$ is $\mu$-Lipschitzian for some $\mu \in\left[0,+\infty\right.$ [. For every integer $k \in\{1, \ldots, K\}$, let $\mathcal{G}_{k}$ be a real 
Hilbert space, let $r_{k} \in \mathcal{G}_{k}$, let $g_{k} \in \Gamma_{0}\left(\mathcal{G}_{k}\right)$, let $\varphi_{k} \in \Gamma_{0}\left(\mathcal{G}_{k}\right)$, and let $L_{k} \in \mathcal{B}\left(\mathcal{H}, \mathcal{G}_{k}\right)$; moreover, if $K_{1}+1 \leqslant k \leqslant K_{2}, \varphi_{k}$ is differentiable on $\mathcal{G}_{k}$ and such that $\nabla \varphi_{k}$ is $\beta_{k}$-Lipschitzian for some $\beta_{k} \in\left[0,+\infty\right.$ [, and, if $K_{2}+1 \leqslant k \leqslant K, \varphi_{k}$ is $1 / \beta_{k}$-strongly convex for some $\left.\beta_{k} \in\right] 0,+\infty$ [. Set $\beta=\max \left\{\mu, \beta_{K_{1}+1}, \ldots, \beta_{K}\right\}+\sqrt{1+\sum_{k=1}^{K}\left\|L_{k}\right\|^{2}}$, and assume that

$$
z \in \operatorname{ran}\left(\partial f+\sum_{k=1}^{K} L_{k}^{*} \circ\left(\partial g_{k} \square \partial \varphi_{k}\right) \circ\left(L_{k} \cdot-r_{k}\right)+\nabla h\right)
$$

and

$$
\left(\forall k \in\left\{1, \ldots, K_{2}\right\}\right) \quad 0 \in \operatorname{sri}\left(\operatorname{dom} g_{k}^{*}-\operatorname{dom} \varphi_{k}^{*}\right) .
$$

Solve the primal problem

$$
\underset{x \in \mathcal{H}}{\operatorname{minimize}} f(x)+\sum_{k=1}^{K}\left(g_{k} \square \varphi_{k}\right)\left(L_{k} x-r_{k}\right)+h(x)-\langle x \mid z\rangle,
$$

together with the dual problem

$$
\underset{v_{1} \in \mathcal{G}_{1}, \ldots, v_{K} \in \mathcal{G}_{K}}{\operatorname{minimize}}\left(f^{*} \square h^{*}\right)\left(z-\sum_{k=1}^{K} L_{k}^{*} v_{k}\right)+\sum_{k=1}^{m}\left(g_{k}^{*}\left(v_{k}\right)+\varphi_{k}^{*}\left(v_{k}\right)+\left\langle v_{k} \mid r_{k}\right\rangle\right) .
$$

Remark 6.2 It follows from (6.2) and [8, Propositions 11.16, 14.15, 15.7(i), and 24.27] that

$$
(\forall k \in\{1, \ldots, K\}) \quad g_{k} \square \varphi_{k} \in \Gamma_{0}\left(\mathcal{G}_{k}\right) \quad \text { and } \quad \partial g_{k} \square \partial \varphi_{k}=\partial\left(g_{k} \square \varphi_{k}\right) .
$$

Hence, using the same type of arguments as in the proof of Proposition [5.3, we can deduce similar conditions for (6.1) to hold, e.g., that (6.3) have a solution and that $\left(r_{k}\right)_{1 \leqslant k \leqslant K}$ lie in the strong relative interior of $\left\{\left(L_{k} x-y_{k}\right)_{1 \leqslant k \leqslant K} \mid x \in \operatorname{dom} f\right.$ and $\left.(\forall k \in\{1, \ldots, K\}) y_{k} \in \operatorname{dom} g_{k}+\operatorname{dom} \varphi_{k}\right\}$.

Proposition 6.3 Consider the setting of Problem 6.1. Let $\left(a_{1,1, n}\right)_{n \in \mathbb{N}},\left(b_{1,1, n}\right)_{n \in \mathbb{N}}$, and $\left(c_{1,1, n}\right)_{n \in \mathbb{N}}$ be absolutely summable sequences in $\mathcal{H}$. For every integer $k \in\{1, \ldots, K\}$, let $\left(a_{2, k, n}\right)_{n \in \mathbb{N}},\left(b_{2, k, n}\right)_{n \in \mathbb{N}}$, and $\left(c_{2, k, n}\right)_{n \in \mathbb{N}}$ be absolutely summable sequences in $\mathcal{G}_{k}$; moreover, if $1 \leqslant k \leqslant K_{1}$, let $\left(b_{1, k+1, n}\right)_{n \in \mathbb{N}}$ be an absolutely summable sequence in $\mathcal{G}_{k}$, and, if $K_{1}+1 \leqslant k \leqslant K_{2}$, let $\left(a_{1, k+1, n}\right)_{n \in \mathbb{N}}$ and $\left(c_{1, k+1, n}\right)_{n \in \mathbb{N}}$ be absolutely summable sequences in $\mathcal{G}_{k}$. Let $x_{0} \in \mathcal{H}, y_{1,0} \in \mathcal{G}_{1}, \ldots, y_{K_{2}, 0} \in \mathcal{G}_{K_{2}}, v_{1,0} \in \mathcal{G}_{1}, \ldots$, and 
$v_{K, 0} \in \mathcal{G}_{K}$, let $\left.\varepsilon \in\right] 0,1 /(\beta+1)\left[\right.$, let $\left(\gamma_{n}\right)_{n \in \mathbb{N}}$ be a sequence in $[\varepsilon,(1-\varepsilon) / \beta]$, and set

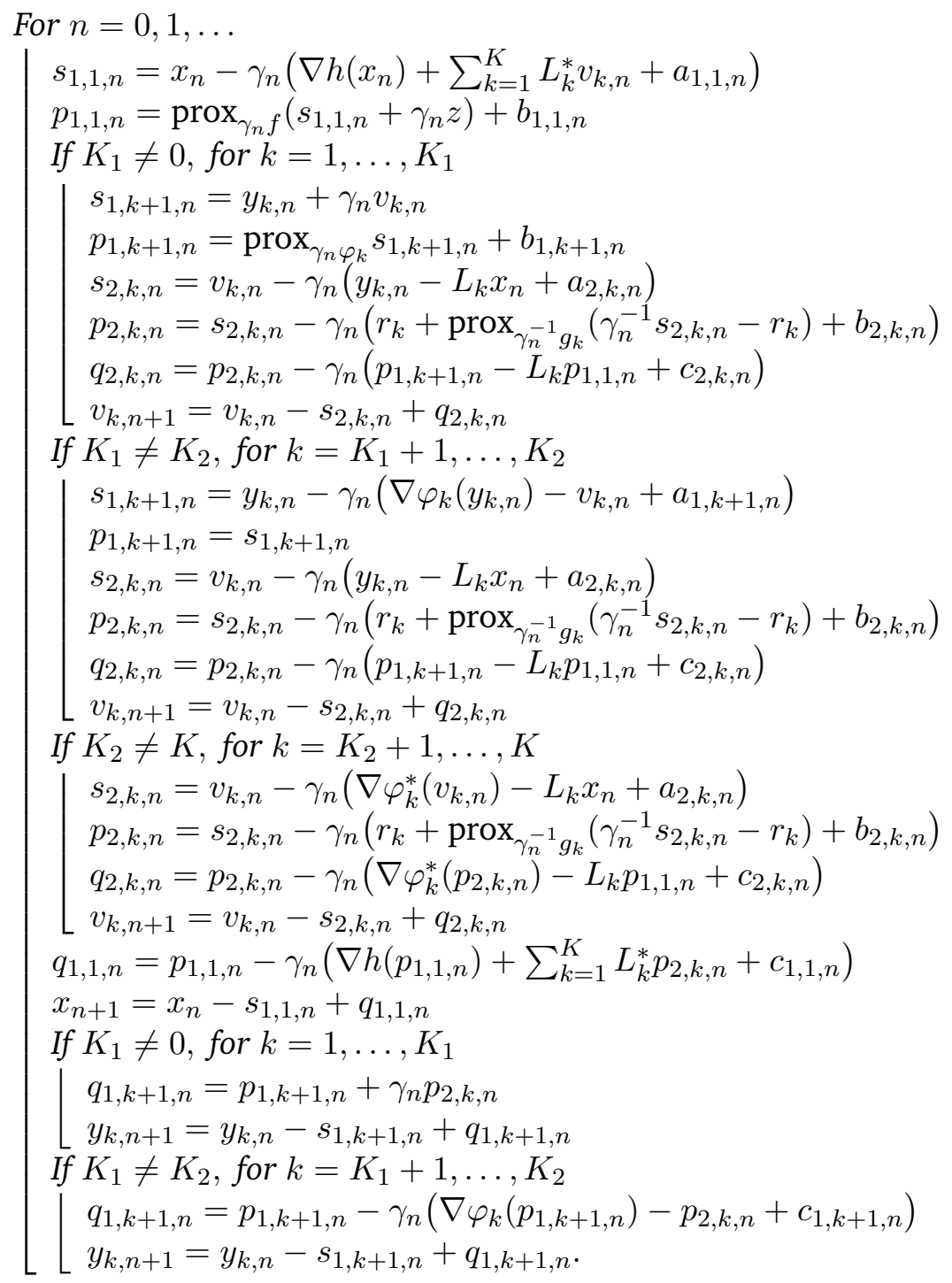

Then the following hold for some solution $\bar{x}$ to (6.3) and some solution $\left(\overline{v_{1}}, \ldots, \overline{v_{K}}\right)$ to (6.4).

(i) $x_{n} \rightarrow \bar{x}$ and $(\forall k \in\{1, \ldots, K\}) v_{k, n} \rightarrow \overline{v_{k}}$.

(ii) Suppose that $f$ or $h$ is uniformly convex at $\bar{x}$. Then $x_{n} \rightarrow \bar{x}$.

(iii) Suppose that, for some $l \in\{1, \ldots, K\}$, $g_{l}^{*}$ is uniformly convex at $\overline{v_{l}}$. Then $v_{l, n} \rightarrow \overline{v_{l}}$.

(iv) Suppose that $K_{2} \neq K$ and that, for some $l \in\left\{K_{2}+1, \ldots, K\right\}$, $\varphi_{l}^{*}$ is uniformly convex at $\overline{v_{l}}$. Then $v_{l, n} \rightarrow \overline{v_{l}}$.

Proof. Using (6.5) and the same arguments as in the proof of Proposition 5.4, we first identify Problem 6.1 as a special case of Problem 3.1 with $A=\partial f, C=\nabla h$, and $(\forall k \in\{1, \ldots, K\}) B_{k}=\partial g_{k}$ and $S_{k}=\partial \varphi_{k}$. Using (1.12), we then deduce the results from Proposition 3.2. $\mathrm{C}$

We conclude this section with an application to the approximation of inconsistent convex feasibility problems where, for the sake of brevity, we discuss only the primal problem. 
Example 6.4 In Problem 6.1, set $K_{1}=K_{2}=K, z=0, h=0, f=0$, and, for every $k \in\{1, \ldots, K\}$ $r_{k}=0$ and $g_{k}=\iota_{C_{k}}$, where $C_{k}$ is a nonempty closed convex subset of $\mathcal{G}_{k}$ with projection operator $P_{k}$. In addition, suppose that

$$
(\forall k \in\{1, \ldots, K\}) \quad \operatorname{Argmin} \varphi_{k}=\{0\}, \varphi_{k}(0)=0, \text { and } 0 \in \operatorname{sri}\left(\operatorname{dom} \iota_{C_{k}}^{*}-\operatorname{dom} \varphi_{k}^{*}\right) .
$$

It follows from [8, Proposition 15.7(i)] that the infimal convolutions $\left(\iota_{C_{k}} \square \varphi_{k}\right)_{1 \leqslant k \leqslant K}$ are exact. Hence, (6.3) becomes

$$
\underset{x \in \mathcal{H}}{\operatorname{minimize}} \sum_{k=1}^{K} \min _{y_{k} \in C_{k}} \varphi_{k}\left(L_{k} x-y_{k}\right),
$$

and it is assumed to have at least one solution. We can interpret (6.8) as a relaxation of the (possibly inconsistent) convex feasibility problem

$$
\text { find } \bar{x} \in \mathcal{H} \text { such that }(\forall k \in\{1, \ldots, K\}) \quad L_{k} \bar{x} \in C_{k} .
$$

Indeed, it follows from (6.7) that, if (6.9) is consistent, then its solutions coincide with those of (6.8). Furthermore, in view of (1.12), Algorithm (6.6) can be written as

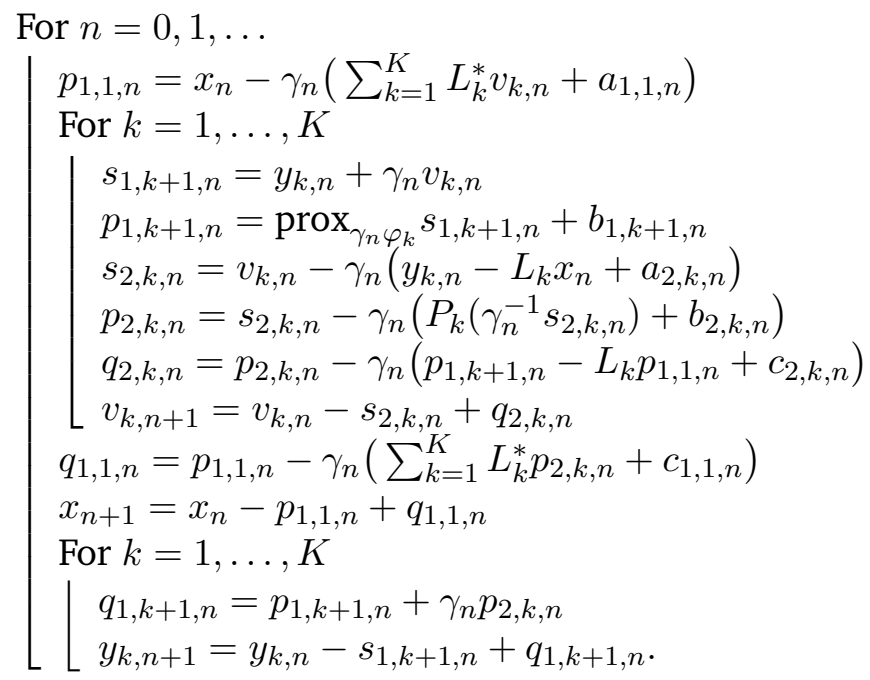

By Proposition 6.3](i), $\left(x_{n}\right)_{n \in \mathbb{N}}$ converges weakly to a solution to (6.8) if $\inf _{n \in \mathbb{N}} \gamma_{n}>0$ and $\sup _{n \in \mathbb{N}} \gamma_{n}<\left(1+\sum_{k=1}^{K}\left\|L_{k}\right\|^{2}\right)^{-1 / 2}$. Now suppose that, for every $k \in\{1, \ldots, K\}, \mathcal{G}_{k}=\mathcal{H}, L_{k}=\operatorname{Id}$, $\varphi_{k}=\iota_{\{0\}}$ if $k=1$, and $\varphi_{k}=\omega_{k}\|\cdot\|^{2}$, where $\left.\omega_{k} \in\right] 0,+\infty[$, if $k \neq 1$. Then (6.9) reduces to the feasibility problem of finding $\bar{x} \in \bigcap_{k=1}^{K} C_{k}$ and (6.8) reduces to the constrained least-squares relaxation studied in [18], namely, $\underset{x \in C_{1}}{\operatorname{minimize}} \sum_{k=2}^{K} \omega_{k} d_{C_{k}}^{2}(x)$.

\section{References}

[1] F. Acker and M. A. Prestel, Convergence d'un schéma de minimisation alternée, Ann. Fac. Sci. Toulouse V. Sér. Math., vol. 2, pp. 1-9, 1980.

[2] H. Attouch, J. Bolte, P. Redont, and A. Soubeyran, Alternating proximal algorithms for weakly coupled convex minimization problems. Applications to dynamical games and PDE's, J. Convex Anal., vol. 15, pp. 485-506, 2008. 
[3] H. Attouch, L. M. Briceño-Arias, and P. L. Combettes, A parallel splitting method for coupled monotone inclusions, SIAM J. Control Optim., vol. 48, pp. 3246-3270, 2010.

[4] H. Attouch, A. Cabot, P. Frankel, and J. Peypouquet, Alternating proximal algorithms for linearly constrained variational inequalities: application to domain decomposition for PDE's, Nonlinear Anal., vol. 74, pp. 7455-7473, 2011.

[5] H. Attouch, P. Redont, and A. Soubeyran, A new class of alternating proximal minimization algorithms with costs-to-move, SIAM J. Optim., vol. 18, pp. 1061-1081, 2007.

[6] F. Bach, R. Jenatton, J. Mairal, and G. Obozinski, Optimization with sparsity-inducing penalties, Found. Trends Machine Learning, vol. 4, pp. 1-106, 2012.

[7] A. B. Bakušinskiĭ and B. T. Polyak, The solution of variational inequalities, Soviet Math. - Doklady, vol. 15, pp. 1705-1710, 1974.

[8] H. H. Bauschke and P. L. Combettes, Convex Analysis and Monotone Operator Theory in Hilbert Spaces. Springer, New York, 2011.

[9] H. H. Bauschke, P. L. Combettes, and D. R. Luke, Finding best approximation pairs relative to two closed convex sets in Hilbert spaces, J. Approx. Theory, vol. 127, pp. 178-192, 2004.

[10] H. H. Bauschke, P. L. Combettes, and S. Reich, The asymptotic behavior of the composition of two resolvents, Nonlinear Anal., vol. 60, pp. 283-301, 2005.

[11] H. Brézis and M. Sibony, Méthodes d'approximation et d'itération pour les opérateurs monotones, Arch. Ration. Mech. Anal., vol. 28, pp. 59-82, 1967/1968.

[12] L. M. Briceño-Arias and P. L. Combettes, Convex variational formulation with smooth coupling for multicomponent signal decomposition and recovery, Numer. Math. Theory Methods Appl., vol. 2, pp. 485-508, 2009.

[13] L. M. Briceño-Arias and P. L. Combettes, A monotone+skew splitting model for composite monotone inclusions in duality, SIAM J. Optim., vol. 21, pp. 1230-1250, 2011.

[14] L. M. Briceño-Arias, P. L. Combettes, J.-C. Pesquet, and N. Pustelnik, Proximal algorithms for multicomponent image recovery problems, J. Math. Imaging Vision, vol. 41, pp. 3-22, 2011.

[15] J.-F. Cai, B. Dong, S. Osher, and Z. Shen, Image restoration: Total variation, wavelet frames, and beyond, J. Amer. Math. Soc., vol. 25, pp. 1033-1089, 2012.

[16] P. L. Combettes, Construction d'un point fixe commun à une famille de contractions fermes, C. R. Acad. Sci. Paris Sér. I Math., vol. 320, pp. 1385-1390, 1995.

[17] P. L. Combettes, Solving monotone inclusions via compositions of nonexpansive averaged operators, Optimization, vol. 53, pp. 475-504, 2004.

[18] P. L. Combettes and P. Bondon, Hard-constrained inconsistent signal feasibility problems, IEEE Trans. Signal Process., vol. 47, pp. 2460-2468, 1999.

[19] P. L. Combettes and J.-C. Pesquet, Primal-dual splitting algorithm for solving inclusions with mixtures of composite, Lipschitzian, and parallel-sum type monotone operators, Set-Valued Var. Anal., vol. 20, pp. 307-330, 2012.

[20] P. L. Combettes and V. R. Wajs, Signal recovery by proximal forward-backward splitting, Multiscale Model. Simul., vol. 4, pp. 1168-1200, 2005.

[21] J. Douglas, Jr. and H. H. Rachford, Jr., On the numerical solution of heat conduction problems in two or three space variables, Trans. Amer. Math. Soc., vol. 82, pp. 421-439, 1956.

[22] J. M. Dye and S. Reich, Unrestricted iterations of nonexpansive mappings in Hilbert space, Nonlinear Anal., vol. 18, pp. 199-207, 1992.

[23] J. Eckstein and D. P. Bertsekas, On the Douglas-Rachford splitting method and the proximal point algorithm for maximal monotone operators, Math. Program., vol. 55, pp. 293-318, 1992. 
[24] F. Facchinei and J.-S. Pang, Finite-Dimensional Variational Inequalities and Complementarity Problems. Springer-Verlag, New York, 2003.

[25] P. Frankel, Alternating proximal algorithm with costs-to-move, dual description and application to PDE's, Discrete Contin. Dyn. Syst. Ser. S, vol. 5, pp. 545-557, 2012.

[26] C. F. Gauss, Theoria Motus Corporum Coelestium. Perthes and Besser, Hamburg, 1809.

[27] J. Gilles, Noisy image decomposition: a new structure, texture and noise model based on local adaptivity, J. Math. Imaging Vision, vol. 28, pp. 285-295, 2007.

[28] R. Glowinski and P. Le Tallec, Augmented Lagrangian and Operator-Splitting Methods in Nonlinear Mechanics. SIAM, Philadelphia, 1989.

[29] M. Goldburg and R. J. Marks II, Signal synthesis in the presence of an inconsistent set of constraints, IEEE Trans. Circuits Syst., vol. 32, pp. 647-663, 1985.

[30] G. M. Korpelevich, The extragradient method for finding saddle points and other problems, Matecon, vol. 12, pp. 747-756, 1976.

[31] A. M. Legendre, Nouvelles Méthodes pour la Détermination de l'Orbite des Comètes. Courcier, Paris, 1805.

[32] N. Lehdili and B. Lemaire, The barycentric proximal method, Comm. Appl. Nonlinear Anal., vol. 6, pp. 29-47, 1999.

[33] E. S. Levitin and B. T. Polyak, Constrained minimization methods, U.S.S.R. Comput. Math. Math. Phys., vol. 6, pp. 1-50, 1966.

[34] P. L. Lions and B. Mercier, Splitting algorithms for the sum of two nonlinear operators, SIAM J. Numer. Anal., vol. 16, pp. 964-979, 1979.

[35] P. Mahey and D. T. Pham, Partial regularization of the sum of two maximal monotone operators, RAIRO Modél. Math. Anal. Numér., vol. 27, pp. 375-392, 1993.

[36] B. Mercier, Topics in Finite Element Solution of Elliptic Problems (Lectures on Mathematics, no. 63). Tata Institute of Fundamental Research, Bombay, 1979.

[37] B. Mercier, Inéquations Variationnelles de la Mécanique (Publications Mathématiques d'Orsay, no. 80.01). Université de Paris-XI, Orsay, France, 1980.

[38] S. Setzer, G. Steidl, and T. Teuber, Infimal convolution regularizations with discrete $\ell_{1}$-type functionals, Commun. Math. Sci., vol. 9, pp. 797-827, 2011.

[39] M. Sibony, Méthodes itératives pour les équations et inéquations aux dérivées partielles non linéaires de type monotone, Calcolo, vol. 7, pp. 65-183, 1970.

[40] J. E. Spingarn, Partial inverse of a monotone operator, Appl. Math. Optim., vol. 10, pp. 247-265, 1983.

[41] J.-L. Starck, M. Elad, and D. L. Donoho, Image decomposition via the combination of sparse representations and a variational approach, IEEE Trans. Image Process., vol. 14, pp. 1570-1582, 2005.

[42] P. Tseng, Applications of a splitting algorithm to decomposition in convex programming and variational inequalities, SIAM J. Control Optim., vol. 29, pp. 119-138, 1991.

[43] P. Tseng, A modified forward-backward splitting method for maximal monotone mappings, SIAM J. Control Optim., vol. 38, pp. 431-446, 2000.

[44] R. S. Varga, Matrix Iterative Analysis, 2nd edition. Springer-Verlag, New York, 2000.

[45] B. C. Vũ, A splitting algorithm for dual monotone inclusions involving cocoercive operators, Adv. Comput. Math., to appear. http://www.springerlink. com/content/m177247u22644173/

[46] C. Zălinescu, Convex Analysis in General Vector Spaces, World Scientific, River Edge, NJ, 2002. 